\title{
Monitoring Deformation in Graphene Through Hyperspectral Synchrotron Spectroscopy to Inform Fabrication
}

Winter, Allen; Rojas, Wudmir; Williams, Adrienne D. ; Kim, Steve S.; Ouchen, Fahima ; Fisher, Daniel A.; Weiland, Conan; Principe, Edward; Banerjee, Sarbajit; Huynh, Chuong; Naik, Rajesh R. ; Liu, Yijin; Mehta, Apurva; Grote, James; Predergast, David; Campo, Eva

\section{Journal of Physical Chemistry C}

DOI:

10.1021/acs.jpcc.7b01376

Published: 01/01/2017

Peer reviewed version

Cyswllt i'r cyhoeddiad / Link to publication

Dyfyniad o'r fersiwn a gyhoeddwyd / Citation for published version (APA):

Winter, A., Rojas, W., Williams, A. D., Kim, S. S., Ouchen, F., Fisher, D. A., Weiland, C.,

Principe, E., Banerjee, S., Huynh, C., Naik, R. R., Liu, Y., Mehta, A., Grote, J., Predergast, D., \& Campo, E. (2017). Monitoring Deformation in Graphene Through Hyperspectral Synchrotron Spectroscopy to Inform Fabrication. Journal of Physical Chemistry C, 121(29), 15653-15664. https://doi.org/10.1021/acs.jpcc.7b01376

\footnotetext{
Hawliau Cyffredinol / General rights

Copyright and moral rights for the publications made accessible in the public portal are retained by the authors and/or other copyright owners and it is a condition of accessing publications that users recognise and abide by the legal requirements associated with these rights.

- Users may download and print one copy of any publication from the public portal for the purpose of private study or research.

- You may not further distribute the material or use it for any profit-making activity or commercial gain

- You may freely distribute the URL identifying the publication in the public portal ?
}

Take down policy

If you believe that this document breaches copyright please contact us providing details, and we will remove access to the work immediately and investigate your claim. 
Subscriber access provided by Olson Library | Northern Michigan University

Article

\section{Monitoring Deformation in Graphene Through Hyperspectral Synchrotron Spectroscopy to Inform Fabrication}

Allen Douglas Winter, Wudmir Yudy Rojas, Adrienne D. Williams, Steve S. Kim, Fahima Ouchen, Daniel A Fischer, Conan Weiland, Edward Principe, Sarbajit Banerjee, Chuong Huynh, Rajesh R. Naik, Yijin Liu, Apurva Mehta, James Gerard Grote, David Prendergast, and Eva M. Campo

J. Phys. Chem. C, Just Accepted Manuscript • DOI: 10.1021/acs.jpcc.7b01376 • Publication Date (Web): 30 Jun 2017

Downloaded from http://pubs.acs.org on July 1, 2017

\section{Just Accepted}

"Just Accepted" manuscripts have been peer-reviewed and accepted for publication. They are posted online prior to technical editing, formatting for publication and author proofing. The American Chemical Society provides "Just Accepted" as a free service to the research community to expedite the dissemination of scientific material as soon as possible after acceptance. "Just Accepted" manuscripts appear in full in PDF format accompanied by an HTML abstract. "Just Accepted" manuscripts have been fully peer reviewed, but should not be considered the official version of record. They are accessible to all readers and citable by the Digital Object Identifier (DOI®). "Just Accepted" is an optional service offered to authors. Therefore, the "Just Accepted" Web site may not include all articles that will be published in the journal. After a manuscript is technically edited and formatted, it will be removed from the "Just Accepted" Web site and published as an ASAP article. Note that technical editing may introduce minor changes to the manuscript text and/or graphics which could affect content, and all legal disclaimers and ethical guidelines that apply to the journal pertain. ACS cannot be held responsible for errors or consequences arising from the use of information contained in these "Just Accepted" manuscripts. 


\title{
Monitoring Deformation in Graphene Through
}

\section{Hyperspectral Synchrotron Spectroscopy to Inform}

\section{Fabrication}

\begin{abstract}
Allen Douglas Winter ${ }^{1}$, Wudmir Y. Rojas ${ }^{1}$, Adrienne D. Williams ${ }^{2}$, Steve S. Kim ${ }^{2}$, Fahima
Ouchen $^{2}$, Daniel A. Fischer ${ }^{3}$ Conan Weiland ${ }^{4}$, Edward Principe $^{4}$, Sarbajit Banerjee ${ }^{5}$, Chuong
\end{abstract}

Huynh ${ }^{6}$, Rajesh R. Naik ${ }^{2}$, Yijin Liu ${ }^{7}$, Apurva Mehta ${ }^{7}$, James Grote $^{2}$, David Prendergast ${ }^{8}$, and Eva

M. Campo*1, 9

${ }^{1}$ School of Electronic Engineering, Bangor University, Bangor, LL571UT UK

${ }^{2}$ Materials and Manufacturing Directorate, Air Force Research Laboratory, Wright-Patterson, AFB, Fairborn, OH 45433, USA

${ }^{3}$ National Institute of Standards and Technology, Gaithersburg, MD 20899, USA

${ }^{4}$ Synchrotron Research Inc, Melbourne, FL 32901, USA

${ }^{5}$ Department of Chemistry, Texas A\&M University, College Station, TX 77840, USA

${ }^{6}$ Carl Zeiss Microscopy, LLC One Corporation Way Peabody, MA 01960

${ }^{7}$ Stanford Synchrotron Radiation Laboratory, Stanford University, SLAC, Stanford, California, 94309 


\author{
${ }^{8}$ Molecular Foundry, Lawrence Berkeley National Laboratory, Berkeley, CA 94720, USA \\ ${ }^{9}$ Department of Physics and Astronomy, University of Texas at San Antonio, TX 78249, USA
}

\begin{abstract}
The promise from graphene to produce devices with high mobilities and detectors with fast response times is truncated in practice by strain and deformation originating during growth and subsequent processing. This work describes effects from graphene growth, multiple layer transfer, and substrate termination on out of plane deformation, critical to device performance. Synchrotron spectroscopy data was acquired with a state-of-the-art hyperspectral large-area detector to describe growth and processing with molecular sensitivity at wafer lengthscales. A study of methodologies used in data analysis discouraged dichroic ratio approaches in favor of orbital vector approximations and data mining algorithms. Orbital vector methods provide a physical insight into mobility-detrimental rippling by identifying ripple frequency as main actor, rather than intensity; which was confirmed by data mining algorithms, and in good agreement with electron scattering theories of corrugation in graphene. This work paves the way to efficient information from mechanical properties in graphene in a high throughput mode throughout growth and processing in a Materials by Design approach.
\end{abstract}

\title{
INTRODUCTION
}

The remarkable properties of graphene have attracted vast attention from both academia and industry, making the material a prime candidate for applications in optoelectronics, sensors, and 
flexible electronic devices, amongst others. ${ }^{1-4}$ On these lines, recent progress in 2D materials beyond graphene is generating expectation to improve optical modulators and advanced terahertz communications in the realm of photonics. ${ }^{5-7}$ Regardless of the application space, the maturity of graphene technologies is still at early stages. Indeed, although high-quality graphene can be epitaxially grown on silicon carbide (SiC) substrates, high costs limit mass production. ${ }^{8}$ Further, adequate flexibility on fabrication paradigms needs of transferred graphene from the initial growth substrate, typically $\mathrm{SiC}$ or copper $(\mathrm{Cu})$, while maintaining its pristine properties. ${ }^{9}$ It is therefore critical to characterize graphene systems produced via affordable, mass-production prone methods such as chemical vapor deposition (CVD), which are low-cost and better support this transfer process. ${ }^{10,11}$ At present, theoretical properties of graphene are far from being reached experimentally due to defects and perturbations in the pristine $\mathrm{sp}^{2}$ structure. ${ }^{12}$ It is well established that out-of-plane deformation (i.e. rippling) and in-plane strain in graphene are initially introduced during high-temperature growth. ${ }^{13,14}$ Upon cooling, mismatch in thermal expansion coefficient between graphene and growth substrate produces a compressed structure, ${ }^{13}$ which is stabilized through the nucleation of intrinsic ripples and strain. ${ }^{15}{ }^{16}$ Post-growth processing of graphene systems can introduce further in-plane strain upon transfer onto nonnative substrates and increase deformation. ${ }^{16-19}$ These effects are of crucial importance, in particular for electronic applications, as strain fluctuations limit carrier mobility in detriment to operational devices, ${ }^{14}$ promoting the need for reliable characterization techniques. Clearly, substrates play a role in the amount of strain in transferred graphene, either in plane or derived from out-of-plane deformation. The importance of substrate effects has been highlighted earlier. ${ }^{14}$ Substrate effects are attributed to imperfect adhesion during transfer and overall deformation on the substrate that will induce forces on the atoms in the graphitic structure, which 
results in strain and deformation. With all, the overall strain that transferred graphene is under is inflicted through a variety of mechanisms, from the transfer process itself - often in harsh environments - to defects and impurities on the substrate, and even from incommensuration between graphene and substrate. ${ }^{14}$

In this work, we apply a Materials by Design approach to graphene CVD fabrication and subsequent transfer to address fabrication-induced out of plane deformation effects in a quantitative fashion. ${ }^{20}$ In this scheme, we will make use of $a b$ initio calculations to produce a mathematical graphene standard that will be compared to five experimental systems: epitaxiallygrown graphene on $\mathrm{SiC}$, as well as single and four monolayers graphene grown via $\mathrm{CVD}$ on $\mathrm{Cu}$, and subsequently transferred to both silicon- and carbon-terminated SiC (SLG/SiC-Si, SLG/SiCC, 4LG/SiC-Si, 4LG/SiC-C). This experimental design will establish a baseline in two realms; first with respect to the theoretical standard, and second with respect to the epitaxially grown graphene on $\mathrm{SiC}$, hence justifying the selection of these substrates. Multiple layer graphene is an additional variable here, known to enhance conductivity, ${ }^{21}$ where a multiplicity of stacking geometries enables tailoring of band structures. ${ }^{22}$ In this scenario, initially nucleated deformation might propagate throughout the graphene stack or further relax depending on subsequent substrate interactions.

\section{EXPERIMENT \\ Angle-resolved hyperspectral NEXAFS spectroscopy \\ The synchrotron technique of choice is Near Edge X-Ray Fine Structure (NEXAFS) spectroscopy, ${ }^{23}$ which has been used to study chemical and electronic structure of a wide range of systems, from organic semiconductors, ${ }^{24}$ to polymer composites; ${ }^{25,}{ }^{26}$ NEXAFS has also}


proven a valuable tool for the quantitative determination of molecular orientation. ${ }^{27-29}$ In particular, the ability of this technique to measure molecular orientation makes it ideal in the study of graphene deformations. ${ }^{10}$ The value brought forward by monitoring $\pi^{*}$ intensity orientations rests upon the notion that out-of-plane deformations perturb the original orientation and distribution of the orbitals. ${ }^{17,26}$ Additionally, although not analyzed in the present study, inplane strain in graphene can also be characterized with NEXAFS, as changes in bond lengths generate energy shifts in the $\sigma^{*}$ resonance. $^{27}$

Notably, the high sensitivity of NEXAFS spectroscopy can now be deployed to large areas (at the mm scale) with resolutions at the micron-scale, due to the advent of hyperspectral large area detectors such as the large-area rapid imaging analysis tool (LARIAT, Synchrotron Research Inc.) used in this work (Figure 1a). ${ }^{30,31}$ Details about the NEXAFS detector are provided in the Supporting Information. This development will prove essential towards informing industrial graphene manufacturing. ${ }^{11,} 20$ Clearly, stacking and NEXAFS measurements of multiple transferred layers will add to the complexity ingrained in single layer effects. With all, a parametric quantification of the overall out-of-plane deformation will be possible through specifically designed data analytics, developed in this work. On those lines, fast acquisition of hyperspectral files results in large data sets-systems imaged at every energy interval (Figure 1b), yielding a spectrum corresponding to each pixel—which need of specific data treatment towards adequate exploitation, i.e. data reduction towards information extraction yielding an outof-plane deformation parameter. To this end, we critically evaluate analytical data methodologies in NEXAFS spectroscopy to ultimately identify and quantify out of plane deformation effects at wafer lengthscales with molecular sensitivity. 
a)

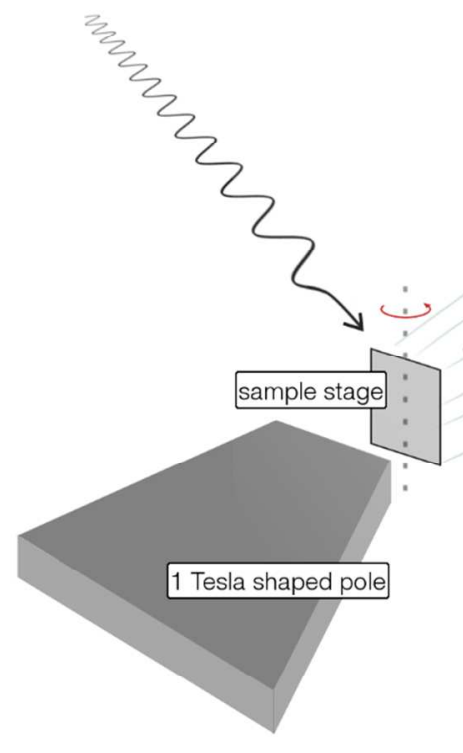

c)
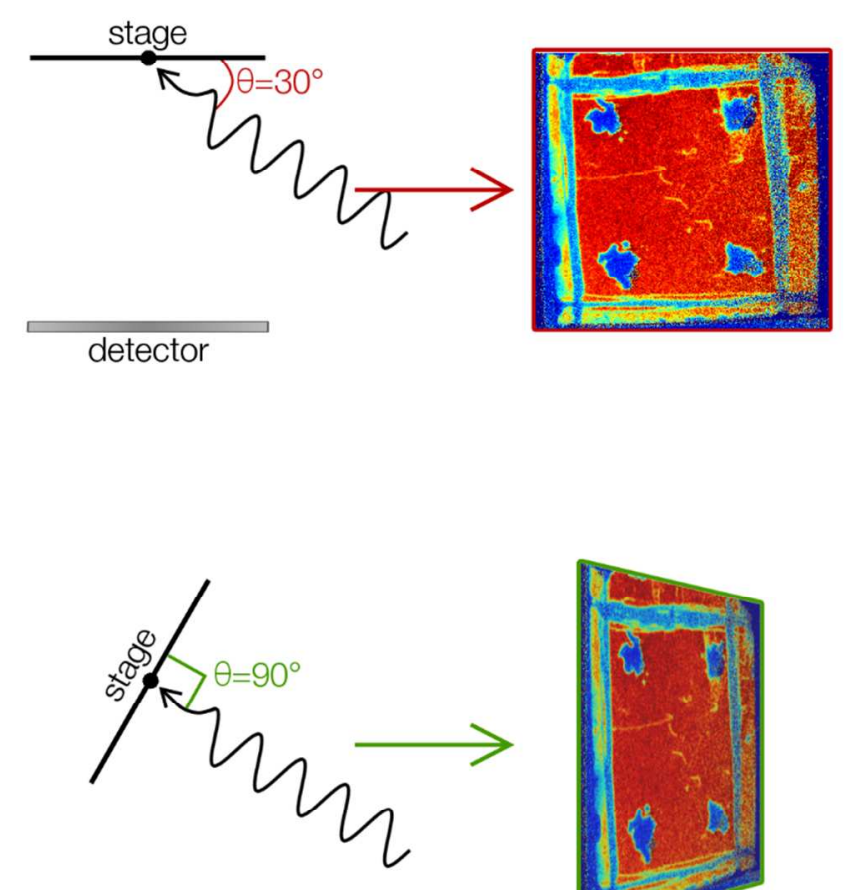

detector

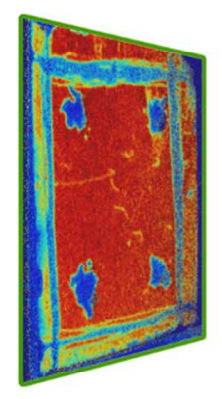

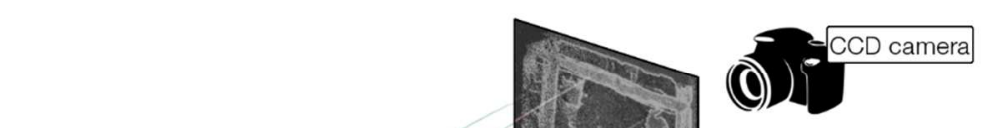

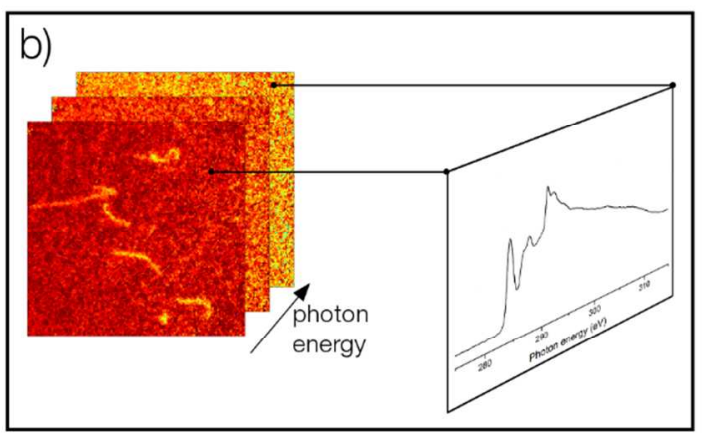

d)
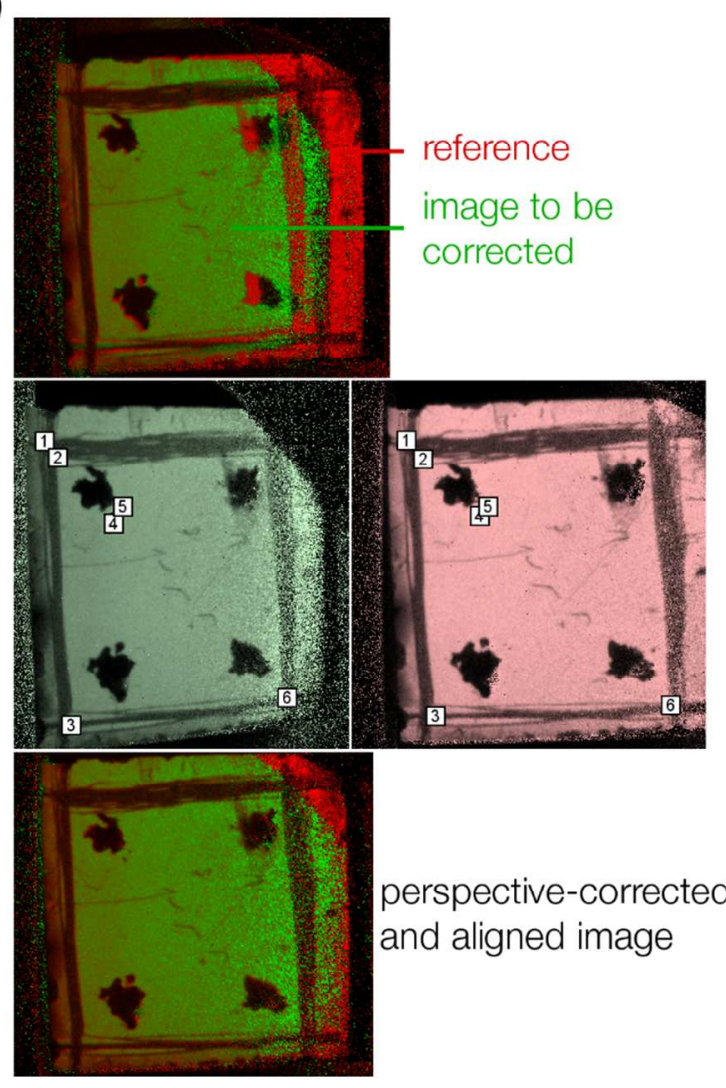

perspective-corrected and aligned image 
Figure 1. Acquisition of hyperspectral NEXAFS image stacks. a) The sample is placed within a uniform $1 \mathrm{~T}$ magnetic field which confines electron trajectories. The photon beam is wobbled up and down to provide full-field illumination for each incident photon energy. Emitted Auger electrons travel along the magnetic field lines, through a high-pass filter and a channel plate. Generated electron pulses are impinged on a phosphor-coated fibre-optic feedthrough, which is imaged with a CCD camera. b) The sample (here, epitaxially-grown graphene) is imaged at each photon energy interval - each pixel in the stack represents an individual spectrum. c) Tilting the stage to acquire NEXAFS at various angles results in spatially-uncorrelated stacks. d) Stacks from different angular acquisitions are manually corrected by correlating pixels to a reference using the LDF software.

The ability to collect data at various incidence angles by tilting the sample stage offers a unique opportunity to study the spatial distribution of out-of-plane deformation in angle-resolved images (Figure 1c). ${ }^{17}$ For hyperspectral detection, perspective distortions in images resulting from stage tilting need to be corrected in order to perform accurate angular characterization of particular ROIs. This is achieved by fiducial mark alignment in images throughout all angular acquisitions (Figure 1d), which is performed by the Lariat Data Figure (LDF) software (Synchrotron Research Inc.). The procedure results in a hyperspectral energy-angle superstack: a spatially-linked series of hyperspectral energy stacks (or 'stacks' for short) for every system (Figure 2a). In each superstack, we ensure that spectra are extracted from identical areas throughout angular acquisitions (Figure 2b). 
a)

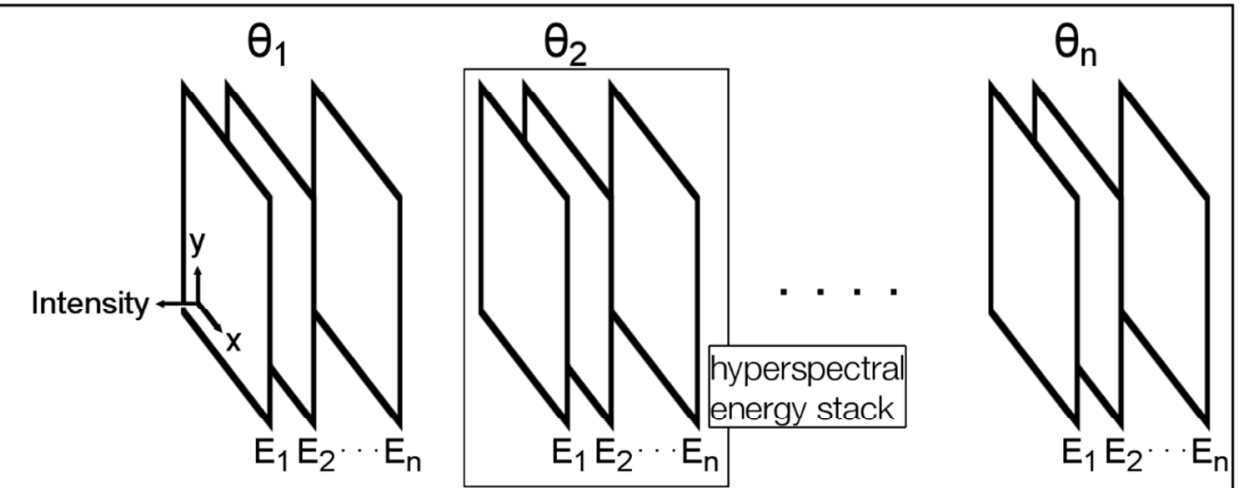

hyperspectral energy-angle superstack

b)

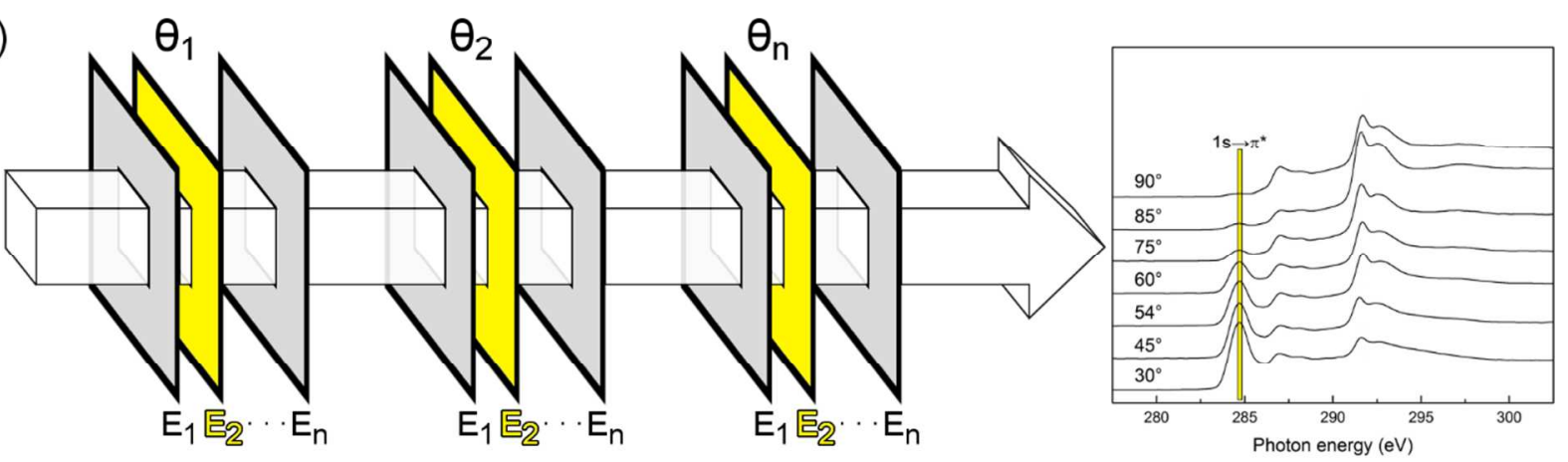

C)
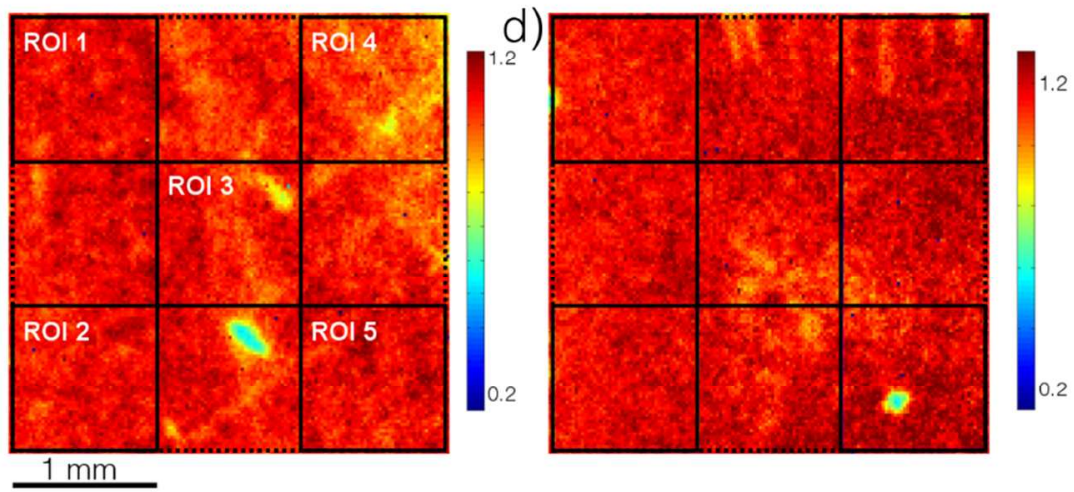

e)
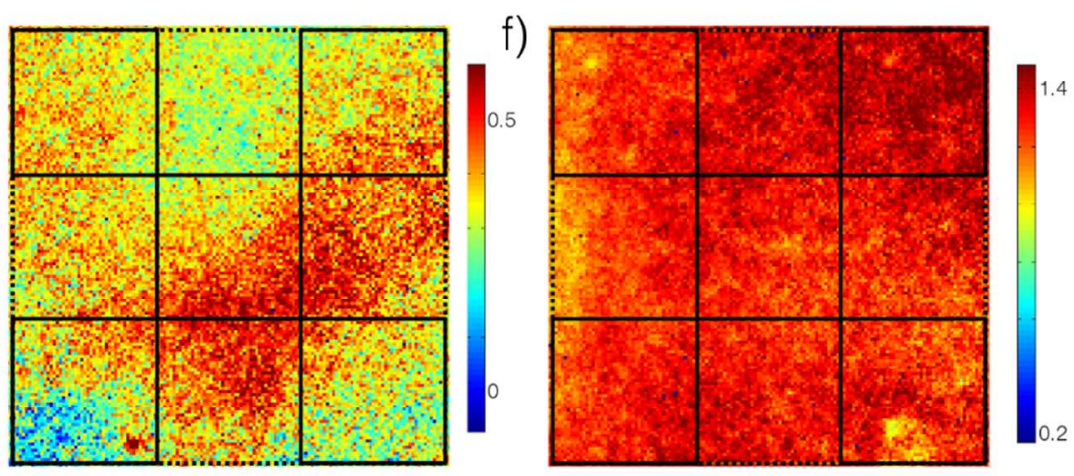
Figure 2. Alignment and perspective correction of experimental data to enable precise ROI correlation between angular acquisitions, and NEXAFS scans of graphene systems under study. a) Post-correction, a hyperspectral energy-angle superstack is assembled for each experimental system, consisting of a hyperspectral energy stack for every acquisition angle. Superstacks are thus functions of spatial $\mathrm{x}$ and $\mathrm{y}$ coordinates, photon energy, intensity, and acquisition angle. b) Schematic representation of data extraction: angular spectra acquired from a precise area in a graphene system, with energy E2 representing the $\pi^{*}$ resonance. c) $\left.\mathrm{SLG} / \mathrm{SiC}-\mathrm{Si}, \mathrm{d}\right) 4 \mathrm{LG} / \mathrm{SiC}-\mathrm{Si}$, e) $\mathrm{SLG} / \mathrm{SiC}-\mathrm{C}$, f) 4LG/SiC-C. Images tuned around $284.7 \mathrm{eV}$, and acquired at $30^{\circ}$, rendering intense $\pi^{*}$ resonances. ROIs are $1 \mathrm{~mm} \times 1 \mathrm{~mm}$, and each pixel is $\sim 40 \mu \mathrm{m}$.

\section{EXPERIMENTAL SECTION}

\section{Synthesis}

Graphene monolayers were grown on Copper foil by chemical vapor deposition (CVD) method in a tube furnace (OTF-1200x-STM, MTI Corp, CA) equipped with a scroll vacuum pump. Whilst the $\mathrm{Cu}$ foil was heated up to $1000{ }^{\circ} \mathrm{C}$, hydrogen gas was injected at 125 mTorr for $30 \mathrm{~min}$, followed by injection of methane gas at 1.25 Torr for a further $30 \mathrm{~min}$; the furnace was then cooled down to room temperature while the flow of hydrogen and methane remained.

Transfer onto SiC substrates was achieved using a thermal release tape (Nitto Americas Inc., $\mathrm{CA}$ ). The tape was placed over the graphene-coated $\mathrm{Cu}$ foil, followed by oxygen plasma treatment for $5 \mathrm{~min}$. The ensemble was then etched in $\left(\mathrm{NH}_{4}\right)_{2} \mathrm{~S}_{2} \mathrm{O}_{8}$ solution for $2 \mathrm{hr}$, rinsed with deionized water, and dried with $\mathrm{N}_{2}$. The $\mathrm{SiC} /$ graphene/thermal release tape was then heated at $125{ }^{\circ} \mathrm{C}$ to release single monolayers onto the substrate. Following this procedure, graphene single monolayer / four monolayers were transferred onto both Si- and C-terminated SiC. 
Epitaxial graphene was grown on commercial nominally on-axis oriented $6 \mathrm{H}-\mathrm{SiC}(0001)$ (SiCrystal AG, Germany). Prior to epitaxy, surface polishing damage was removed through etching in hydrogen for $15 \mathrm{~min}$. at 1 bar and $1550{ }^{\circ} \mathrm{C}$. Epitaxial growth was performed in a vertical cold-wall reactor with a double-walled, water-cooled quartz tube and a graphite susceptor in a slow flow of argon (purity 5.0). Heating and cooling rates were $2-3 \circ \mathrm{C}$ per second. Typical annealing time was $15 \mathrm{~min}$.

\section{Characterization}

For Helium Ion Microscopy characterization, untreated CVD-grown graphene was transferred to a $\mathrm{SiN}_{\mathrm{x}}$ grid and imaged in an ORION Plus He-IM Zeiss at $35 \mathrm{kV}$ with the assistance of a flood gun to prevent surface charging.

Hyperspectral near-edge X-ray absorption fine structure (NEXAFS) data were collected at NIST beamline U7A, located at the National Synchrotron Light Source, Brookhaven National Laboratory, using a large area rapid imaging analytical tool (LARIAT, Synchrotron Research Inc.). Spectra were recorded at the Carbon K-edge in partial electron yield mode. By tilting the sample, acquisitions for each system were made at $30^{\circ}, 45^{\circ}, 50^{\circ}, 54^{\circ}, 60^{\circ}, 85^{\circ}$, and $90^{\circ}$ incidence of the X-rays to the plane of the sample. A full field electron spectrometer produces a stack of two-dimensional NEXAFS images; full spectra can then be extracted from individual pixels.

Hyperspectral images were angle-corrected using the LDF software (Synchrotron Research Inc.) to enable spectra extraction at various incidence angles from corresponding areas in each graphene system. Spectra normalisation was performed on the same software. Five $1 \mathrm{~mm}$ x 1 
$\mathrm{mm}$ regions of interest (ROIs) were analysed in each system, as well as the enclosing $3 \mathrm{~mm} \times 3$ mm ROI.

\section{COMPUTATIONAL DETAILS}

Free-standing graphene primitive unit cell of 2 carbon atoms with lattice parameter of $2.465 \AA$ was relaxed by using plane-wave density functional theory (DFT) under the general-gradient approximation (GGA) of Perdue-Burke-Ernzerhof (PBE) exchange-correlation functional implemented in Quantum ESPRESSO suite within the pseudopotential approximation. ${ }^{32-34} \mathrm{We}$ employed ultrasoft pseudopotentials (C.pbe-van_ak.UPF), energy cutoff of 30 Ry for plane wave basis set and 300 Ry for charge density, a k-sampling grid in the Monkhorst-Pack scheme of $30 \times 30 \times 1$, total energy tolerance of $10-6$ and vacuum of $\sim 20 \AA$ along the Z-axis to avoid interactions between periodic images. Atomic forces at equilibrium position were converged below the $0.01 \mathrm{eV} / \AA$ threshold.

Our input to perform NEXAFS spectra simulations is a periodic 10x10 supercell size containing 200 carbon atoms. Ab initio NEXAFS spectroscopy calculations at C K-edge were modelled in the framework of the excited electron and core-hole $(\mathrm{XCH})$ approximation, ${ }^{35}$ and spectra were projected for angles of incidence of $0^{\circ}, 10^{\circ}, 20^{\circ}, 30^{\circ}, 35^{\circ}, 40^{\circ}, 45^{\circ}, 54^{\circ}, 60^{\circ}, 65^{\circ}$, $75^{\circ}, 85^{\circ}$ and 90 . In order to simulate the excitation of the carbon atom due to the X-ray, one electron from the 1s level of the carbon pseudopotential has been removed. One carbon atom from the supercell has been used to calculate the unoccupied states under the pseudopotential approximation that includes the core-hole interactions. XCH-NEXAFS used the PBE form for the exchange-correlation potential within GGA approximation. 


\section{RESULTS AND DISCUSSION}

In this study, hyperspectral NEXAFS maps of epitaxial graphene (Figure 1b), as well as SLG/SiC-Si, SLG/SiC-C, 4LG/SiC-Si, and 4LG/SiC-C (Figure 2c-f) were acquired at various incidence angles, following the procedure highlighted in Figure 2a. Images Fig 2c-f correspond to the $1 \mathrm{~s} \rightarrow \pi^{*}$ transitions around $284.7 \mathrm{eV}$, taken from the $30^{\circ}$ stacks, where these features show highest $\pi^{*}$ intensities for flat graphene. ${ }^{36}$ Full extracted experimental spectra from Fig 2c-f (transferred graphene) and Fig 1b (epitaxial graphene) as well as the theoretical standard spectra, are shown in Figure 3. Angular intensity trends are shown in Fig 3, which will be investigated towards out-of-plane deformation analysis. Confirming predominantly flat graphene, $\pi{ }^{*} \mathrm{C}=\mathrm{C}$ resonance intensities (at $284.7 \mathrm{eV}$ ) are minimal when the incoming beam is at $90^{\circ}$ to the substrate, and decrease with incidence angle, whilst $\sigma^{*}{ }_{\mathrm{C}-\mathrm{C}}$ resonance intensities $($ at $\sim 291 \mathrm{eV}$ ) exhibit the opposite trend. ${ }^{36}$ Due to the low variability on the $\pi^{*} \mathrm{C}=\mathrm{C}$ intensities, SLG/SiC-C is not considered in our data analysis - this, and additional spectral resonances in each sample, are described in more detail in the Supporting Information. 

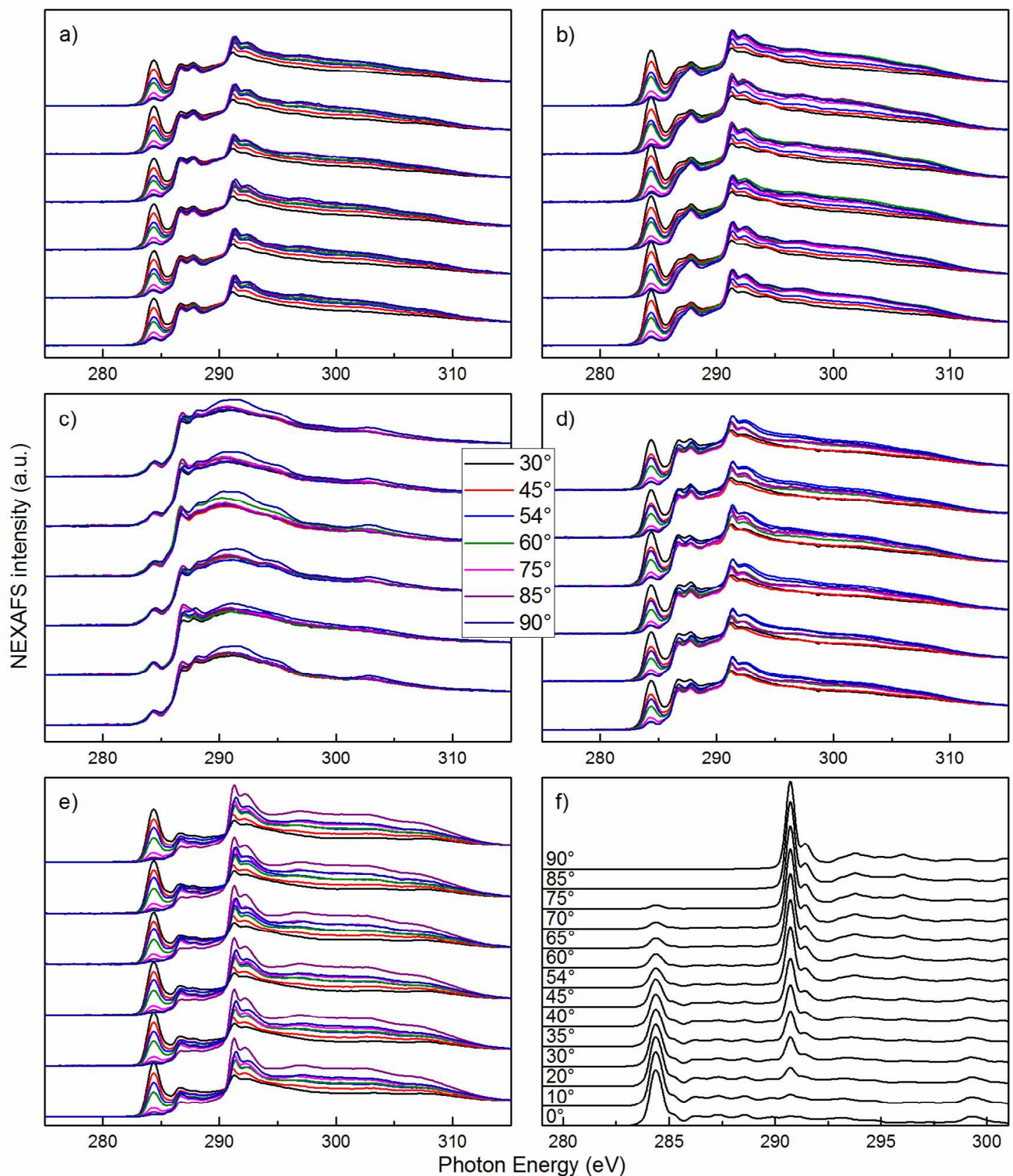

Figure 3. Extracted angle-resolved Carbon K-edge NEXAFS spectra. a) SLG/SiC-Si, b) 4LG/SiC-Si, c) SLG/SiC-C, d) 4LG/SiC-C, e) epitaxial graphene, f) Theoretical freestanding graphene. Resonances described in detail in the Supporting Information.

For the analysis of out of plane deformation it is necessary to establish a baseline which excludes all angular-independent contributions. ${ }^{28}$ To this end, angle-resolved $a b$ initio NEXAFS 
spectra of pristine graphene were computed. A top level workflow describing calculations is shown in Figure 4a. Briefly, the excited state is treated through constrained Density Functional Theory (DFT) following the excited electron and core hole $(\mathrm{XCH})$ approach implemented in the Shirley code. ${ }^{35}$ The transitions are calculated using Fermi's Golden Rule. Angular projections are performed according to the geometric coordinates defined in Figure $4 \mathrm{~b}$.

a)

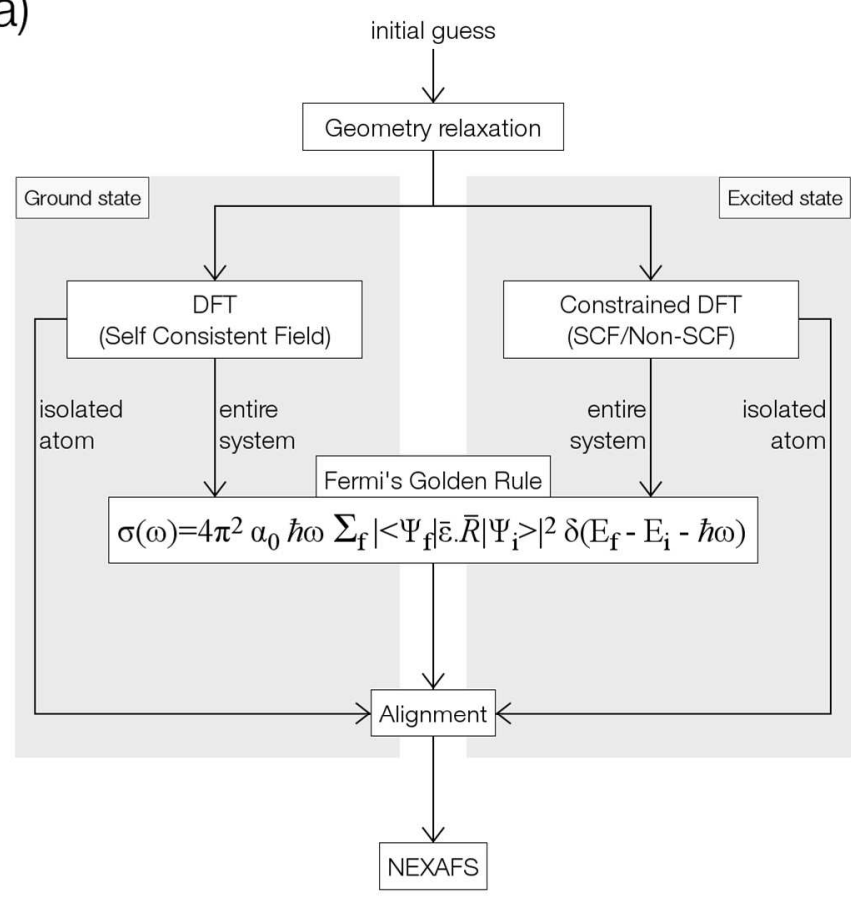

b)

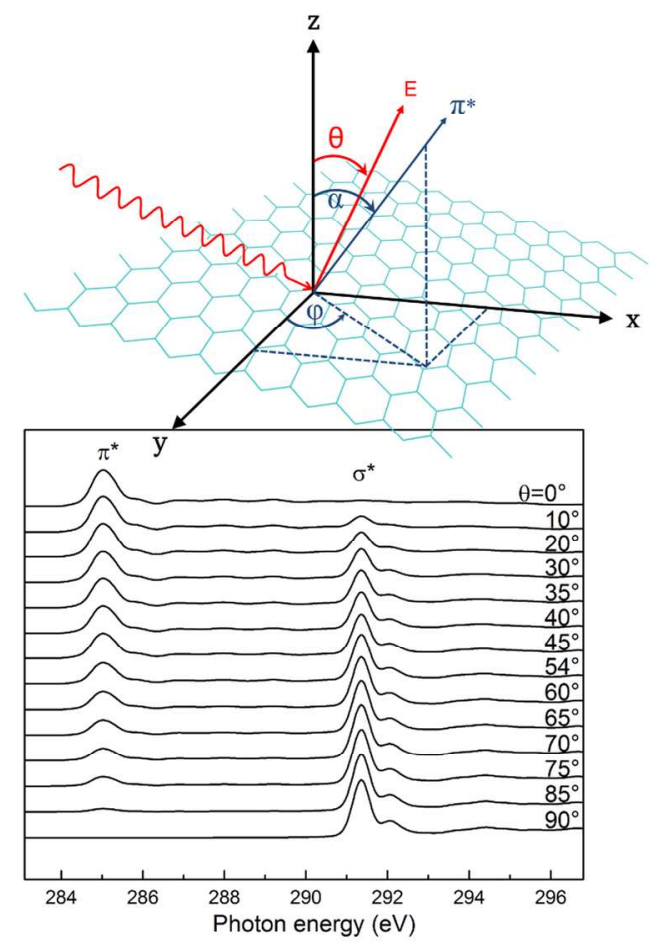

c)

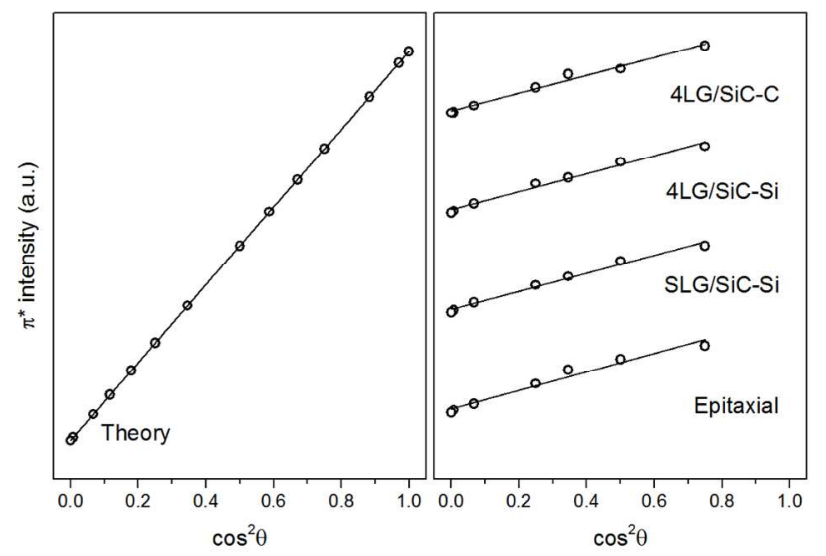

d)

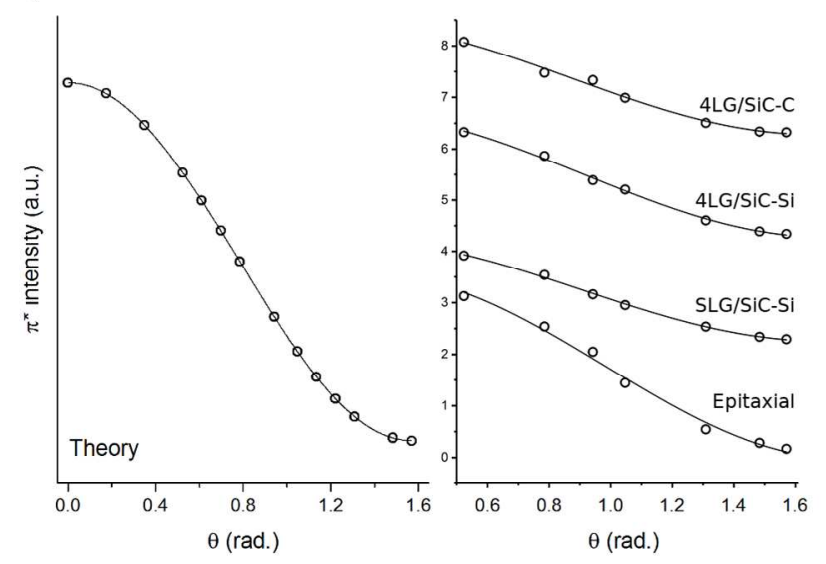


Figure 4. Theoretical angular-resolved NEXAFS calculation algorithm, and $\pi^{*}$ and $\sigma^{*}$ intensities plotted against $\cos ^{2} \theta$ and $\sin ^{2} \theta$ respectively for both theoretical and experimental graphene systems. a) Following geometry optimization to find the most energetically stable structure, the ground state is computed through standard DFT, whilst the excited state is computed through constrained DFT following the excited electron and core hole $(\mathrm{XCH})$ approximation. NEXAFS spectra are calculated using Fermi's Golden Rule. b) Spectra are then projected for various beam polar angles $(\theta)$; geometric definitions also show $\pi^{*}$ vector described by angles $\alpha$ and $\varphi$, as used in the orbital vector approximation. c) Dichroic ratios of theoretical graphene, epitaxial graphene, SLG/SiC-Si, 4LG/SiC-Si and 4LG/SiC-C computed from $3 \mathrm{~mm} x$ $3 \mathrm{~mm}$ ROIs are $-1,-0.93,-0.94,-0.94$, and -0.97 , respectively. Full results presented in Table S1, Supporting Information. d) $\pi^{*}$ NEXAFS intensities fitted with Eq. 2. Fitting parameter $\alpha$ from 3 $\mathrm{mm} \times 3 \mathrm{~mm}$ ROIs resulted in 0 for theoretical graphene, 11.8 for epitaxial graphene/SiC, 7.2 for SLG/SiC-Si, 7.9 for 4LG/SiC-Si, and 5.5 for 4LG/SiC-C. Full results are presented in Table I. 


\section{Dichroic ratio}

A common approach for semi-quantitatively identifying out-of-plane deformation in graphene is through the dichroic ratio (DR), which compares intensities at orthogonal acquisition angles, and is computed following Equation 1. 17, 37, 38

$$
D R=\frac{\left(I_{90^{\circ}}-I_{0^{\circ}}\right)}{\left(I_{90^{\circ}}+I_{0^{\circ}}\right)}
$$

This approach can be applied to both $\pi^{*}$ and $\sigma^{*}$ intensities, as they follow linear and orthogonal trends with $\cos ^{2} \theta$ and $\sin ^{2} \theta$. Analysis of $\sigma^{*}$ intensities in graphene is complex, however, as carbonaceous impurities adsorbed on experimental systems also contribute to the spectral fingerprint. Thus, $\pi^{*}$ intensities are used here. As described by Equation $1, \pi^{*}$ intensities will render $\mathrm{DR}=-1$ for a system with $\pi^{*}$ orbitals perfectly oriented normal to the plane (as in pristine graphene) and $\mathrm{DR}=0$ when orbitals are isotropically arranged.

Fitted $\pi^{*}$ intensities of theoretical graphene and experimental spectra extracted from $3 \mathrm{~mm} \times 3$ mm ROIs are shown in Figure 4c. Theoretical standard confirms a DR of -1 for pristine graphene, as expected. Results (Table S1, Supporting Information) indicate that in this instance epitaxial graphene has the largest deformation, yielding the lowest numerical value of DR, while 4LG/SiC-C has the least deformation, with the highest numerical value of $\mathrm{DR}$, followed by both SLG/SiC-Si and 4LG/SiC-Si, rendering identical DRs. The difference in ratios between all systems (and ROIs within systems) is low, however, suggesting this method suffers from poor molecular sensitivity at these high extents of substrate alignment. Indeed, every ROI in $\mathrm{SLG} / \mathrm{SiC}-\mathrm{Si}$ resulted in $\mathrm{DR}=-0.94$, while clear differences can be observed in the $\pi^{*}$ maps for that system in Figure 2c. Being insensitive to fine out of plane deformation at the length-scale under study, the dichroic ratio can only provide a descriptive indication of relative $\pi^{*}$ orbital alignment, and the method is therefore unsuitable for comprehensive analytics at wafer level. 


\section{Orbital vector approximation}

Following the procedure first outlined by Stöhr, ${ }^{27}$ orientation and alignment of small molecules has been quantitatively determined by classing $\pi^{*}$ and $\sigma^{*}$ orbitals as either vectors or planes, according to their geometry. ${ }^{28,39,40}$ In this model, $\pi^{*}$ orbitals in graphene are classed as vectors normal to the plane. NEXAFS intensities of these orbitals are a function of acquisition angle $\theta$ which — in a system with threefold or higher symmetry_-follow Equation 2, where $\alpha$ is the polar angle of the vector-like orbital, as depicted in Figure $4,{ }^{27}$ and the azimuth $\phi$ is absent in the expression due to symmetry considerations. Indeed, a single sheet of graphene will result in one single orientation with respect to the substrate upon transfer. In an initial approximation, ignoring local perturbations from rippling that will be modelled through $\alpha$, the azimuth angle is chosen to be zero. In addition, the parameter $\mathrm{c}$ was included to account for $\pi^{*}$ intensity variations in highly delocalized systems which combine with additional signals provided by adjacent unperturbed areas.

$\mathrm{I}(\theta)=\mathrm{c}+\mathrm{A}\left(\cos ^{2} \theta \cos ^{2} \alpha+\sin ^{2} \theta \sin ^{2} \alpha+2 \sin \alpha \cos \alpha \sin \theta \cos \theta\right)$

In 2D materials like graphene, out-of-plane deformation produces perturbed orbitals affected throughout multiple atoms in a typically asymmetric rippling configuration, as demonstrated in the Helium Ion micrograph (Figure S3a-b, Supporting Information). We have used DFT approaches to calculate the NEXAFS spectra of individual atoms along an asymmetric ripple. Under this method, such deformations produce non-zero $\alpha$ and $\mathrm{c}$ values resulting from local rippling (Table I and Figure S3c-d Supporting Information), even though the graphene is predominantly flat on the substrate. Since NEXAFS cannot distinguish between local disorder and molecular tilting, the $\alpha$ and c values extracted here embed information related to both homogeneity and magnitude of corrugations. These findings suggest that the orbital vector 
approximation is promising in the characterization of rippling effects in graphene, where $\alpha$ and c are providing physical interpretation on frequency and intensity of rippling phenomena, as discussed below.

Equation 2 was fitted in OriginPro 9, using the Levenberg-Marquardt algorithm. Figure 4d shows $\pi^{*}$ intensities of theoretical graphene, which, as expected, produces $\alpha=c=0$. Experimental systems fitted with Eq. 2 are also shown in Figure 4d. Earlier studies have shown that small errors systematically introduced when extracting $\pi$ *intensities from NEXAFS spectra can have important consequences on the resulting $\alpha$ values. ${ }^{41}$ However, the $\alpha$ and c values obtained from different ROIs form distinct clusters for each experimental graphene system (Table I). Resulting $\alpha$ parameters from each analyzed ROI are depicted in Figure 5. 
Table I. Results of orbital vector approximation method, showing the results of the orbital vector approximation method, described by equation 2 . Here, $\square *$ intensities from representative $3 \mathrm{~mm}$ ROI from each system and the vector approximation fits are shown in Figure $4 \mathrm{~d}$.

\begin{tabular}{|c|c|c|c|c|c|c|c|c|c|}
\hline System & ROI & $\alpha$ (deg.) & $\begin{array}{c}\text { R- } \\
\text { Squared }\end{array}$ & mean & s.d. & c & R-Squared & mean & s.d. \\
\hline $\begin{array}{l}\text { Theoretical } \\
\text { graphene }\end{array}$ & -- & 0 & 1 & 0 & 0 & 0 & 1 & 0 & 0 \\
\hline \multirow{6}{*}{ Epitaxial } & 1 & 10.8 & 0.9943 & \multirow{6}{*}{11.66} & \multirow{5}{*}{1.27} & -0.035 & 0.9942 & \multirow{6}{*}{-0.0366} & \multirow{6}{*}{0.0120} \\
\hline & 2 & 10 & 0.9936 & & & 0.006 & 0.9936 & & \\
\hline & 3 & 11.8 & 0.9909 & & & -0.037 & 0.9908 & & \\
\hline & 4 & 12.8 & 0.9896 & & & -0.063 & 0.9896 & & \\
\hline & 5 & 12.9 & 0.9904 & & & -0.054 & 0.9904 & & \\
\hline & $3 \mathrm{~mm}$ & 11.3 & 0.9927 & & \multirow{7}{*}{0.87} & -0.028 & 0.9927 & & \\
\hline \multirow{6}{*}{ SLG/SiC-Si } & 1 & 8.1 & 0.999 & \multirow{6}{*}{7.19} & & 0.239 & 0.9990 & \multirow{6}{*}{0.2520} & \multirow{6}{*}{0.0086} \\
\hline & 2 & 8 & 0.9983 & & & 0.248 & 0.9983 & & \\
\hline & 3 & 6.9 & 0.9986 & & & 0.260 & 0.9987 & & \\
\hline & 4 & 6 & 0.9992 & & & 0.254 & 0.9992 & & \\
\hline & 5 & 6.9 & 0.9998 & & & 0.259 & 0.9999 & & \\
\hline & $3 \mathrm{~mm}$ & 7.1 & 0.9983 & & & 0.255 & 0.9983 & & \\
\hline \multirow{6}{*}{ 4LG/SiC-Si } & 1 & 8.1 & 0.997 & \multirow{6}{*}{7.61} & \multirow{6}{*}{0.59} & 0.253 & 0.9970 & \multirow{6}{*}{0.2883} & \multirow{6}{*}{0.0282} \\
\hline & 2 & 7.6 & 0.9967 & & & 0.269 & 0.9967 & & \\
\hline & 3 & 6.7 & 0.9981 & & & 0.325 & 0.9981 & & \\
\hline & 4 & 8.1 & 0.9975 & & & 0.303 & 0.9975 & & \\
\hline & 5 & 7.6 & 0.9974 & & & 0.291 & 0.9974 & & \\
\hline & $3 \mathrm{~mm}$ & 7.8 & 0.9979 & & & 0.275 & 0.9978 & & \\
\hline \multirow{6}{*}{ 4LG/SiC-C } & 1 & 4.4 & 0.9954 & & & 0.293 & 0.9954 & \multirow{6}{*}{0.2852} & \multirow{6}{*}{0.0222} \\
\hline & 2 & 4.4 & 0.9942 & & & 0.274 & 0.9942 & & \\
\hline & 3 & 6.4 & 0.9884 & 5.63 & 1.09 & 0.278 & 0.9884 & & \\
\hline & 4 & 6.3 & 0.9916 & & & 0.320 & 0.9916 & & \\
\hline & 5 & 6.6 & 0.9881 & & & 0.261 & 0.9881 & & \\
\hline & $3 \mathrm{~mm}$ & 5.4 & 0.9917 & & & 0.281 & 0.9917 & & \\
\hline
\end{tabular}


In fact, Figure 5 also shows a data treatment routine suitable for incorporation in large area substrates and high throughput industrial environments. For optimal speed, not only in data acquisition but also in data processing, it is paramount that reductive analytic techniques are designed and implemented. Here we propose the analysis of selected $1 \mathrm{~mm}$ x $1 \mathrm{~mm}$ ROIs to be compared to the analysis of overall $3 \mathrm{~mm}$ x $3 \mathrm{~mm}$ ROIs (Figure 5a). In this scheme, zero-level data reduction (integration) represents the capabilities of standard, non-hyperspectral NEXAFS spectroscopy, where all the bulk data is being analyzed, hence taxing manufacturing decision making turnaround. Along these lines, the corrugation map (Figure 5b) has been constructed from $\alpha$ values resulting from analysis of $3 \mathrm{~mm} \times 3 \mathrm{~mm}$ ROIs i.e. the whole scanned area. This zero-level data reduction (integration) indicates that, at this length scale, and in agreement with the dichroic ratio, epitaxial graphene is the system with the most out of plane deformation, with 4LG/SiC-Si, SLG/SiC-Si and 4LG/SiC-C decreasing $\alpha$, indicating less corrugation. In contrast to the dichroic ratio, however, this method proves to be significantly more sensitive to out-of-plane deformation variations. And in fact, despite the goodness of fit for both parameters, as shown in the R-squared values, this sensitivity seems enhanced for $\alpha$ versus c variations. Indeed, only meaningful variation arises from comparing the c values between epitaxial and transferred, as shown in Table I. Similarly to the treatment applied to the $\alpha$ parameter, individual $\mathrm{c}$ values are provided for the 5 ROIs in each sample, from where mean and standard deviation are calculated. Data in Table I shows that amongst the transferred systems, mean c values overlap within the brackets of their standard variation, as seen in Table I, and only a net variation is observed between the lower c values of epitaxial and the higher c values from transferred systems, as a 

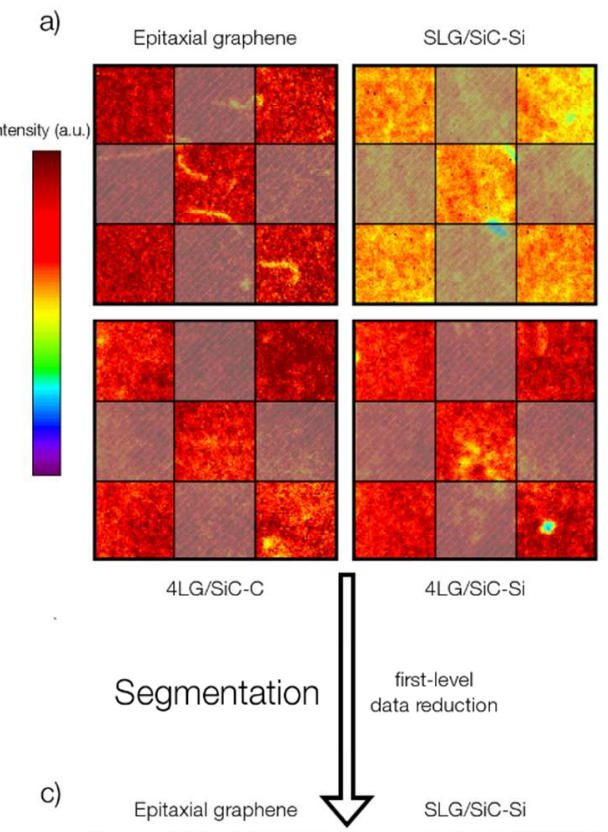

4LG/SiC-Si
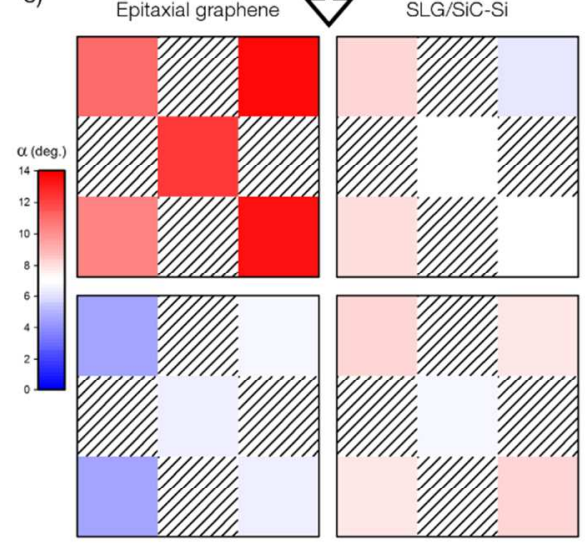

4LG/SiC-C

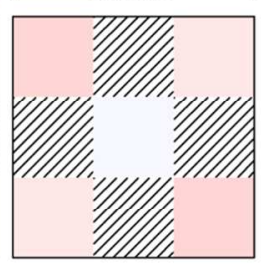

4LG/SiC-Si
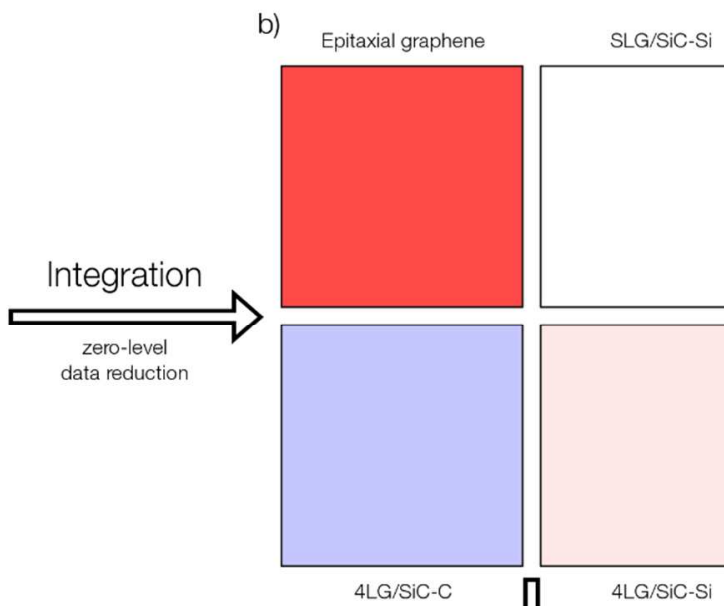

Validation

d)

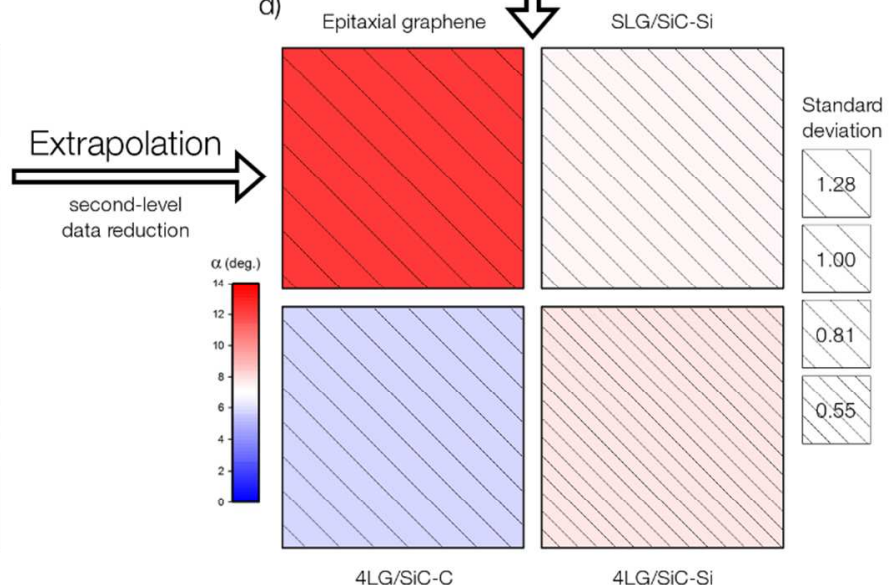

Figure 5. $\alpha$ maps constructed from results of orbital vector approximation. a) NEXAFS $\pi^{*}$ intensities, b) as from $3 \mathrm{~mm}$ x $3 \mathrm{~mm}$ ROIs (zero-level data reduction), c) $\alpha$ s from ROIs 1-5 (first-level data reduction), d) mean $\alpha$ s and standard deviations from ROIs 1-5 (second-level data reduction). Intensity scale in a) is 0-1.9 for epitaxial graphene/SiC, and 0-1.4 for the other three systems. 
In Figure 5c, we have only considered $\alpha$ values resulting from analysis of $1 \mathrm{~mm} \times 1 \mathrm{~mm}$ ROIs. In this scenario, we are only sampling a portion of the overall available data (non-shaded areas in Figure 5a). Values of $\alpha$ in ROIs 1-5 in each sample (Figure 5c) represent the first-level data reduction (segmentation), consistent with the data discussed in ROIs 1-5 in Table I. Segmentation here reveals a significantly increased sensitivity to local out-of-plane deformation variations compared to the dichroic ratio, most pronounced in SLG/SiC-Si, where every ROI produced the same DR, but different values of $\alpha$.

In the second-level data reduction, extrapolation (Figure 5d), the segmented analysis from Figure $5 \mathrm{c}$ is extrapolated from the selected $1 \mathrm{~mm} \times 1 \mathrm{~mm}$ ROIs to the overall $3 \mathrm{~mm} \times 3 \mathrm{~mm}$ ROI. By comparing Figures $5 b$ and $d$, we conclude that the data segmentation strategy has successfully minimized data load whilst still informing on the overall $3 \mathrm{~mm}$ x $3 \mathrm{~mm}$ ROI (validation). Mean $\alpha$ s and standard deviations were computed from $\alpha$ values at ROIs 1-5, and they are also shown in Figure $5 \mathrm{~d}$.

The representations in Figure 5 clearly show a trade-off in terms of choice of target substrate: in absolute terms, 4LG/SiC-C is the system under least corrugation but shows the least homogeneity across the wafer; conversely, SLG/SiC-Si and 4LG/SiC-Si show more corrugation, but show the most homogeneous deformation distribution. The dynamics of growth on each $\mathrm{SiC}$ termination are well documented $;^{9}$ here we highlight the importance of substrate termination upon graphene transfer, with a homogenous but higher out-of-plane deformation level for Sitermination on a four-layer transfer.

As mentioned above, the meaning of $\alpha$ as measured in graphene $\pi^{*}$ intensities is complex. Initially, $\alpha$ had been derived as the overall angle in a molecular collective; such as LangmuirBlodgett monolayers. ${ }^{28}$ In the present scenario, $\alpha$ is revealing combined information of local flat 
areas intertwined with ripples, therefore informing of frequency and intensity of such events in some fashion. Clearly, a lower $\alpha$ is desired, as demonstrated by theoretical graphene. But what phenomena will increase this value? We believe that in fact, a larger $\alpha$ in graphene is indicative of higher frequency of out-of-plane deformation, rather than the intensity of such events, i.e. the size of ripples or of macroscopic scratches from processing. Indeed, scratches observed in ROI 3 and 5 in epitaxial graphene don't have a large impact in the overall $\alpha$ as compared with ROI 2 , with less frequent scratches, as seen in Figure 5c. This is further explored in the next section.

\section{Density-based spatial clustering of applications with noise (DBSCAN)}

The previous sections described the analysis of angular-dependent $\pi^{*}$ intensities within integrated ROIs two orders of magnitude larger than the detector resolution. This final section describes unsupervised analysis of larger graphene areas sampled at the pixel level (40 um) using Density-based spatial clustering of applications with noise (DBSCAN) clustering algorithm. ${ }^{42}$ DBSCAN is, by now, a well-established algorithm. An important advantage of DBSCAN is the minimal a piori knowledge of the system required and its robustness to noise. Liu and coworkers successfully employed this data-mining technique to hard X-ray XANES of $\mathrm{Nd}_{2} \mathrm{Fe}_{14} \mathrm{~B} .{ }^{43}$ Here, we proactively apply DBSCAN to soft X-ray hyperspectral NEXAFS images to cluster based on pixel $\pi^{*}$ intensities acquired at $30^{\circ}$. In this way, out of the two resulting clusters, the one with highest $\pi^{*}$ intensities indicates the most pristine graphene. Prior to clustering, image stacks were cropped and normalised using TXM Wizard. ${ }^{44}$ DBSCAN maps and spectra of resulting clusters are shown in Figure 6. 
$\mathrm{SLG} / \mathrm{SiC}-\mathrm{Si}$
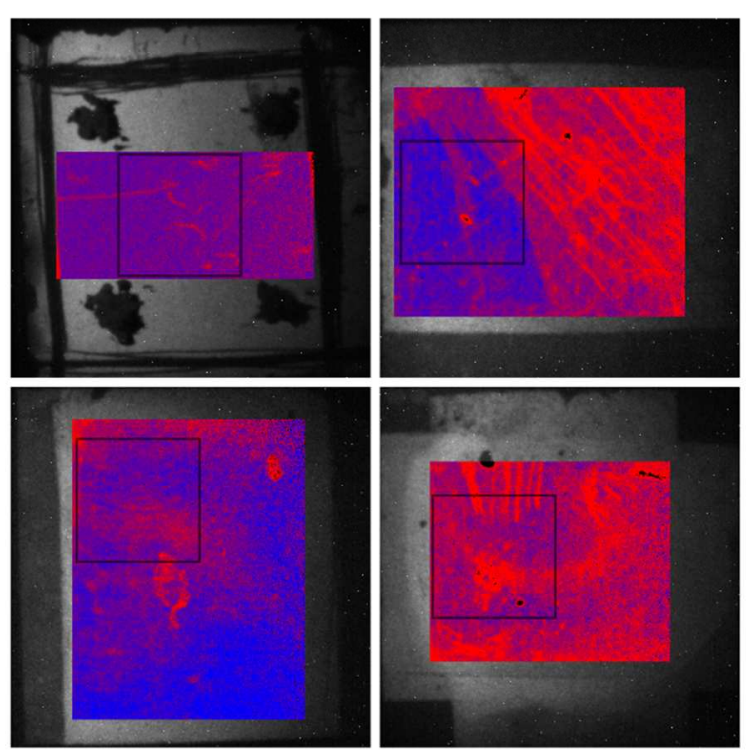

4LG/SiC-C

4LG/SiC-Si

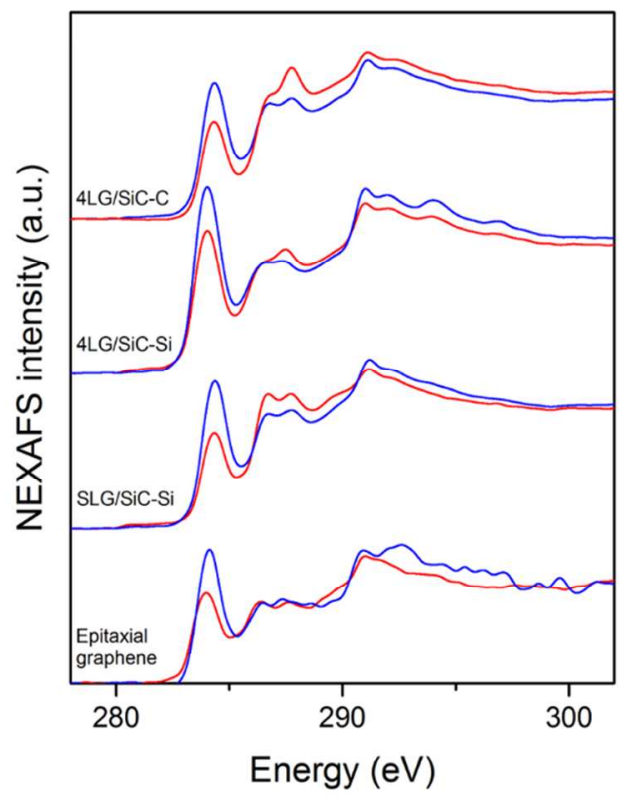

Figure 6. DBSCAN clustering based on $\pi^{*}$ intensity. Cluster maps indicate $4 \mathrm{LG} / \mathrm{SiC}-\mathrm{C}$ has the most pristine graphene. Maps also suggest two distinct length scales of out-of-plane deformations. Black squares indicate the location of $3 \mathrm{~mm} \times 3 \mathrm{~mm}$ ROIs analyzed in the previous sections.

Some features in epitaxial graphene (Figure 6) are likely to be scratches-caused by processing and handling - which resulted in local changes in orientation, hence producing $\pi^{*}$ resonances of lower intensities. For the most part, epitaxial graphene shows a dense mixture of both clusters, suggesting the physical variations (ripples) in the graphene have lenghtscales below the detector resolution $(40 \mu \mathrm{m})$ and that they are very frequent. ${ }^{13,19}$ Indeed, since each pixel is assigned a fraction weight of each cluster, each pixel is rated higher or lower quality depending on the frequency of sub-pixel defects. In contrast to the transferred systems, there are no pure areas consisting of only one cluster: consistent with findings from dichroic ratio and orbital vector approximation methods. This further demonstrates that in this scenario, epitaxial 
graphene is the least pristine graphene in terms of rippling. Indeed, epitaxially-grown graphene suffered from out of plane deformation originating during the cooling-off stage of hightemperature growth. In addition, single-atomic layers typically grow on imperfect $\mathrm{SiC}$ substrates containing many terraces of a $0.5 \mathrm{~nm}$ typical step-height. ${ }^{8}$ During growth, graphene conforms to these terraces, deforming the $\pi$ structures which directly lower electron mobility. ${ }^{8}$

Cluster maps of transferred systems on Si termination show less spotty, well-defined phases. Within the red clusters, lines are seen in a uniform arrangement, revealing ripples of $\sim 100 \mu \mathrm{m}$ in size, formed due to either imperfect graphene-substrate contact during transfer, or to water drain channels during etching and rinsing. ${ }^{19,} 45$ Though the maps are dominated by the red cluster showing least pristine graphene, the clusters are well segregated, suggesting local homogeneity (unlike in epitaxial graphene). For this reason, large-scale ripples are therefore less likely to negatively impact mobilities than the nanometer-scale ripples from epitaxial growth. Further, the contrast in ripple size between epitaxial and transferred graphene leads to the hypothesis that it is frequency of ripples, rather than ripple magnitude, that contributed to $\alpha$ in the orbital vector approximation method. On a similar note, given the reverse behavior of the c parameter, where c values increase with the level of processing, and since both $\mathrm{c}$ and $\alpha$ parameters provide in some measure information on ripple magnitude and frequency; data suggest that the c parameter could be preferentially informing on ripple magnitude.

DBSCAN maps suggest 4LG/SiC-C is the most pristine graphene system in terms of out-ofplane deformation. Indeed, the map of this system is dominated by the blue cluster, with the highest $\pi^{*}$ intensity. Further, the ripples seen in SLG/SiC-Si and 4LG/SiC-Si do not appear in 4LG/SiC-C. These observations are in agreement with findings from the prior methods, where 4LG/SiC-C resulted in both the lowest dichroic ratio, and lowest $\alpha$. In this scenario, epitaxial 
graphene/SiC showing the highest $\alpha$ and lowest $\mathrm{c}$ suggests that either indeed ripple frequency is a dominant mode versus ripple magnitude or that growth was suboptimal.

In fact, according to electron scattering studies, ${ }^{12}$ thermally-created ripples, i.e. nanometer size, have long-range scattering character, ${ }^{15}$ highly impacting carrier mobility in graphene. However, substrate-induced corrugations i.e. up to microns in size imply a short-range scattering potential, ${ }^{19}$ and consequently are less impactful to carrier mobilities. These findings complete the structure-property description of $\alpha$, that follows the same dependence as mobility on defect frequency rather than size, and therefore becomes a good candidate to correlate deformation and performance at wafer scale with molecular sensitivity.

\section{CONCLUSIONS}

Summarizing, the work described here critically examined data treatment methods for characterization of out of plane deformation in graphene, critical to mobilities, through the combination of experimental hyperspectral NEXAFS data and ab-initio calculations. Though all methodologies agree that epitaxial graphene is the most defective system and 4LG/SiC-C the most pristine, the orbital vector approximation method-used here to analyze a large crystal system for the first time-proved significantly more sensitive to fine-scale corrugation. DBSCAN confirmed the results and revealed different deformation length-scales, suggesting values of $\alpha$ in the orbital vector approximation are more influenced by frequency of defects rather than by their magnitude, in good agreement with the reported phenomena behind carrier mobility in graphene. These findings pave the way towards the correlation of out of plane strain 
and carrier mobility through the assessment of the parameter $\alpha$ in a high throughput fashion at wafer scales.

Successful development of the proposed methodology requires characterization of both theoretical and experimental standards to build a library that will allow for adequate discrimination of out-of-plane deformation through a multiplicity of layers, not only in graphene, but in other $2 \mathrm{D}$ systems.

Supporting Information. Full tabulated results and further discussion of Dichroic ratio method and Orbital vector approximation method are available as a Word document.

\section{Corresponding Author}

* e.campo@bangor.ac.uk, evamcampo@gmail.com

\section{Notes}

Certain commercial names are presented in this manuscript for purposes of illustration and do not constitute an endorsement by the National Institute of Standards and Technology.

E. Principe is President at Synchrotron Research, developers of the LARIAT detector used in this work.

\section{ACKNOWLEDGMENTS}

We thank Dr. Chuong Huynh at Carl Zeiss Microscopy for assistance in Helium Ion Microscopy. Project supported by AFOSR Grants FA9550-17-1-0082, FA9550-14-1-0099 and FA9550-15-10289, the U.S. DOE, Office of Science, Office of Basic Energy Sciences, contract number 
DEAC02-98CH10886. Computational work was performed in part through High Performance

Computing Wales project numbers 095 and 224, as part of the HPCW-Project No. 80621, through European Regional Development Funding. Simulations of NEXAFS spectra were executed as part of a User Project at the Molecular Foundry, Lawrence Berkeley National Laboratory, which is supported by the Office of Science, Office of Basic Energy Sciences of the U.S. DOE, contract number DE-AC02-05CH11231.

\section{REFERENCES}

1. Bonaccorso, F.; Sun, Z.; Hasan, T.; Ferrari, A., Graphene photonics and optoelectronics. Nature photonics 2010, 4 (9), 611-622.

2. Novoselov, K. S.; Geim, A. K.; Morozov, S. V.; Jiang, D.; Zhang, Y.; Dubonos, S. V.; Grigorieva, I. V.; Firsov, A. A., Electric field effect in atomically thin carbon films. Science 2004, 306 (5696), 666-669.

3. Schwierz, F., Graphene transistors. Nature nanotechnology 2010, 5 (7), 487-496.

4. $\quad$ Sood, A. K.; Lund, I.; Puri, Y. R.; Efstathiadis, H.; Haldar, P.; Dhar, N. K.; Lewis, J.;

Dubey, M.; Zakar, E.; Wijewarnasuriya, P., Review of graphene technology and its applications for electronic devices. 2015.

5. Sun, Z.; Martinez, A.; Wang, F., Optical modulators with 2D layered materials. Nature Photonics 2016, 10 (4), 227-238.

6. Nagatsuma, T.; Ducournau, G.; Renaud, C. C., Advances in terahertz communications accelerated by photonics. Nature Photonics 2016, 10 (6), 371-379.

7. $\quad$ Won, R.; Xia, F., Flat talk. Nature Photonics 2016, 10 (4), 205-206.

8. Norimatsu, W.; Kusunoki, M., Epitaxial graphene on $\mathrm{SiC}\{0001\}$ : advances and perspectives. Physical Chemistry Chemical Physics 2014, 16 (8), 3501-3511.

9. Tetlow, H.; De Boer, J. P.; Ford, I.; Vvedensky, D.; Coraux, J.; Kantorovich, L., Growth of epitaxial graphene: Theory and experiment. Physics Reports 2014, 542 (3), 195-295.

10. Schultz, B. J.; Patridge, C. J.; Lee, V.; Jaye, C.; Lysaght, P. S.; Smith, C.; Barnett, J.; Fischer, D. A.; Prendergast, D.; Banerjee, S., Imaging local electronic corrugations and doped regions in graphene. Nature communications 2011, 2, 372.

11. Zurutuza, A.; Marinelli, C., Challenges and opportunities in graphene commercialization. Nature nanotechnology 2014, 9 (10), 730-734.

12. Katsnelson, M.; Geim, A., Electron scattering on microscopic corrugations in graphene. Philosophical Transactions of the Royal Society of London A: Mathematical, Physical and Engineering Sciences 2008, 366 (1863), 195-204.

13. Hattab, H.; N’Diaye, A. T.; Wall, D.; Klein, C.; Jnawali, G.; Coraux, J.; Busse, C.; van Gastel, R.; Poelsema, B.; Michely, T., Interplay of wrinkles, strain, and lattice parameter in graphene on iridium. Nano letters 2012, 12 (2), 678-682. 
14. Couto, N. J.; Costanzo, D.; Engels, S.; Ki, D.-K.; Watanabe, K.; Taniguchi, T.; Stampfer, C.; Guinea, F.; Morpurgo, A. F., Random strain fluctuations as dominant disorder source for high-quality on-substrate graphene devices. Physical Review X 2014, 4 (4), 041019.

15. Fasolino, A.; Los, J.; Katsnelson, M. I., Intrinsic ripples in graphene. Nature materials 2007, 6 (11), 858-861.

16. Park, Y.; Choi, J. S.; Choi, T.; Lee, M. J.; Jia, Q.; Park, M.; Lee, H.; Park, B. H., Configuration of ripple domains and their topological defects formed under local mechanical stress on hexagonal monolayer graphene. Scientific reports 2015, 5.

17. Lee, V.; Park, C.; Jaye, C.; Fischer, D. A.; Yu, Q.; Wu, W.; Liu, Z.; Bao, J.; Pei, S.-S.; Smith, C.; Lysaght, P.; Banerjee, S., Substrate hybridization and rippling of graphene evidenced by near-edge X-ray absorption fine structure spectroscopy. The Journal of Physical Chemistry Letters 2010, 1 (8), 1247-1253.

18. Ni, G.-X.; Zheng, Y.; Bae, S.; Kim, H. R.; Pachoud, A.; Kim, Y. S.; Tan, C.-L.; Im, D.; Ahn, J.-H.; Hong, B. H., Quasi-periodic nanoripples in graphene grown by chemical vapor deposition and its impact on charge transport. ACS nano 2012, 6 (2), 1158-1164.

19. Deng, S.; Berry, V., Wrinkled, rippled and crumpled graphene: an overview of formation mechanism, electronic properties, and applications. Materials Today 2016, 19 (4), 197-212.

20. Green, M. L.; Hattrick-Simpers, J. R.; Choi, C. L.; Takeuchi, I.; Joshi, A. M.; Barron, S. C.; Chiang, T.; Davydov, A.; Empedocles, S.; Gregoire, J.; Mehta, A. Fulfilling the Promise of the Materials Genome Initiative with High-Throughput Exprimentation (White Paper); MRS 2014.

21. Kasry, A.; Kuroda, M. A.; Martyna, G. J.; Tulevski, G. S.; Bol, A. A., Chemical doping of large-area stacked graphene films for use as transparent, conducting electrodes. ACS nano 2010, 4 (7), 3839-3844.

22. Latil, S.; Henrard, L., Charge carriers in few-layer graphene films. Physical Review Letters 2006, 97 (3), 036803.

23. Guttmann, P.; Bittencourt, C.; Rehbein, S.; Umek, P.; Ke, X.; Van Tendeloo, G.; Ewels, C. P.; Schneider, G., Nanoscale spectroscopy with polarized X-rays by NEXAFS-TXM. Nature Photonics 2012, 6 (1), 25-29.

24. Opitz, A.; Wilke, A.; Amsalem, P.; Oehzelt, M.; Blum, R.-P.; Rabe, J. P.; Mizokuro, T.; Hörmann, U.; Hansson, R.; Moons, E., Organic heterojunctions: Contact-induced molecular reorientation, interface states, and charge re-distribution. Scientific reports 2016, 6 .

25. Winter, A. D.; Larios, E.; Alamgir, F. M.; Jaye, C.; Fischer, D. A.; Omastova, M.; Campo, E. M., Thermo-active behaviour of ethylene-vinyl acetate $\mid$ multiwall carbon nanotube composites examined by in situ near edge x-ray absorption fine structure spectroscopy. The Journal of Physical Chemistry C 2014, 118 (7), 3733-3741.

26. Winter, A. D.; Czanikova, K.; Larios, E.; Vishniyakov, V.; Jaye, C.; Fischer, D. A.; Omastova, M.; Campo, E. M., Interface dynamics in strained polymer nanocomposites: stick-slip wrapping as a prelude to mechanical backbone twisting derived from sonication-induced amorphization. The Journal of Physical Chemistry C 2015.

27. Stöhr, J., NEXAFS Spectroscopy. Springer: 2003.

28. Outka, D.; Stöhr, J.; Rabe, J.; Swalen, J., The orientation of Langmuir-Blodgett monolayers using NEXAFS. The Journal of chemical physics 1988, 88 (6), 4076-4087.

29. Breuer, T.; Klues, M.; Witte, G., Characterization of orientational order in $\pi$-conjugated molecular thin films by NEXAFS. Journal of Electron Spectroscopy and Related Phenomena 2015, 204, 102-115. 
30. Konicek, A.; Jaye, C.; Hamilton, M.; Sawyer, W.; Fischer, D.; Carpick, R., Near-edge Xray absorption fine structure imaging of spherical and flat counterfaces of ultrananocrystalline diamond tribological contacts: a correlation of surface chemistry and friction. Tribology Letters 2011, 44 (1), 99-106.

31. Baio, J. E.; Jaye, C.; Fischer, D. A.; Weidner, T., Multiplexed Orientation and Structure Analysis by Imaging Near-Edge X-ray Absorption Fine Structure (MOSAIX) for Combinatorial Surface Science. Analytical chemistry 2013, 85 (9), 4307-4310.

32. Kohn, W.; Sham, L. J., Self-consistent equations including exchange and correlation effects. Physical Review 1965, 140 (4A), A1133.

33. Perdew, J. P.; Burke, K.; Ernzerhof, M., Generalized gradient approximation made simple. Physical review letters 1996, 77 (18), 3865.

34. Giannozzi, P.; Baroni, S.; Bonini, N.; Calandra, M.; Car, R.; Cavazzoni, C.; Ceresoli, D.; Chiarotti, G. L.; Cococcioni, M.; Dabo, I., QUANTUM ESPRESSO: a modular and open-source software project for quantum simulations of materials. Journal of Physics: Condensed Matter 2009, 21 (39), 395502.

35. Prendergast, D.; Galli, G., X-Ray Absorption Spectra of Water from First Principles Calculations. Phys. Rev. Lett. 2006, 96 (21), 215502.

36. Schultz, B. J.; Dennis, R. V.; Lee, V.; Banerjee, S., An electronic structure perspective of graphene interfaces. Nanoscale 2014, 6 (7), 3444-3466.

37. Velasco-Velez, J. J.; Wu, C.-H.; Wang, B.-Y.; Sun, Y.; Zhang, Y.; Guo, J.-H.; Salmeron, M., Polarized X-ray Absorption Spectroscopy Observation of Electronic and Structural Changes of Chemical Vapor Deposition Graphene in Contact with Water. The Journal of Physical Chemistry C 2014, 118 (44), 25456-25459.

38. Lee, V.; Whittaker, L.; Jaye, C.; Baroudi, K. M.; Fischer, D. A.; Banerjee, S., Large-area chemically modified graphene films: electrophoretic deposition and characterization by soft Xray absorption spectroscopy. Chemistry of Materials 2009, 21 (16), 3905-3916.

39. Lu, H.; Zeysing, D.; Kind, M.; Terfort, A.; Zharnikov, M., Structure of self-assembled monolayers of partially fluorinated alkanethiols with a fluorocarbon part of variable length on gold substrate. The Journal of Physical Chemistry C 2013, 117 (37), 18967-18979.

40. $\quad$ Frey, S.; Stadler, V.; Heister, K.; Eck, W.; Zharnikov, M.; Grunze, M.; Zeysing, B.; Terfort, A., Structure of thioaromatic self-assembled monolayers on gold and silver. Langmuir 2001, 17 (8), 2408-2415.

41. Peisert, H.; Biswas, I.; Knupfer, M.; Chassé, T., Orientation and electronic properties of phthalocyanines on polycrystalline substrates. physica status solidi (b) 2009, 246 (7), 1529-1545. 42. $\quad$ Ester, M.; Kriegel, H.-P.; Sander, J.; Xu, X. In A density-based algorithm for discovering clusters in large spatial databases with noise, Kdd, 1996; pp 226-231.

43. Duan, X.; Yang, F.; Antono, E.; Yang, W.; Pianetta, P.; Ermon, S.; Mehta, A.; Liu, Y., Unsupervised data mining in nanoscale X-ray spectro-microscopic study of NdFeB magnet. Scientific Reports 2016, 6, 34406.

44. Liu, Y.; Meirer, F.; Williams, P. A.; Wang, J.; Andrews, J. C.; Pianetta, P., TXM-Wizard: a program for advanced data collection and evaluation in full-field transmission X-ray microscopy. Journal of synchrotron radiation 2012, 19 (2), 281-287.

45. Zhu, W.; Low, T.; Perebeinos, V.; Bol, A. A.; Zhu, Y.; Yan, H.; Tersoff, J.; Avouris, P., Structure and electronic transport in graphene wrinkles. Nano letters 2012, 12 (7), 3431-3436. 


\section{TOC graphic}

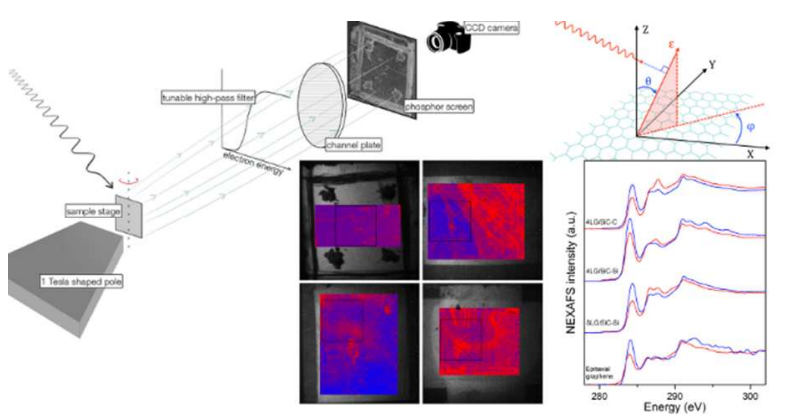

0

1

23

24

25

26

27

29

30

32

33

34

35

36

37

38

39

40

41

42

44

45

46

47

48

49

51

52

53

54

55

56

57 

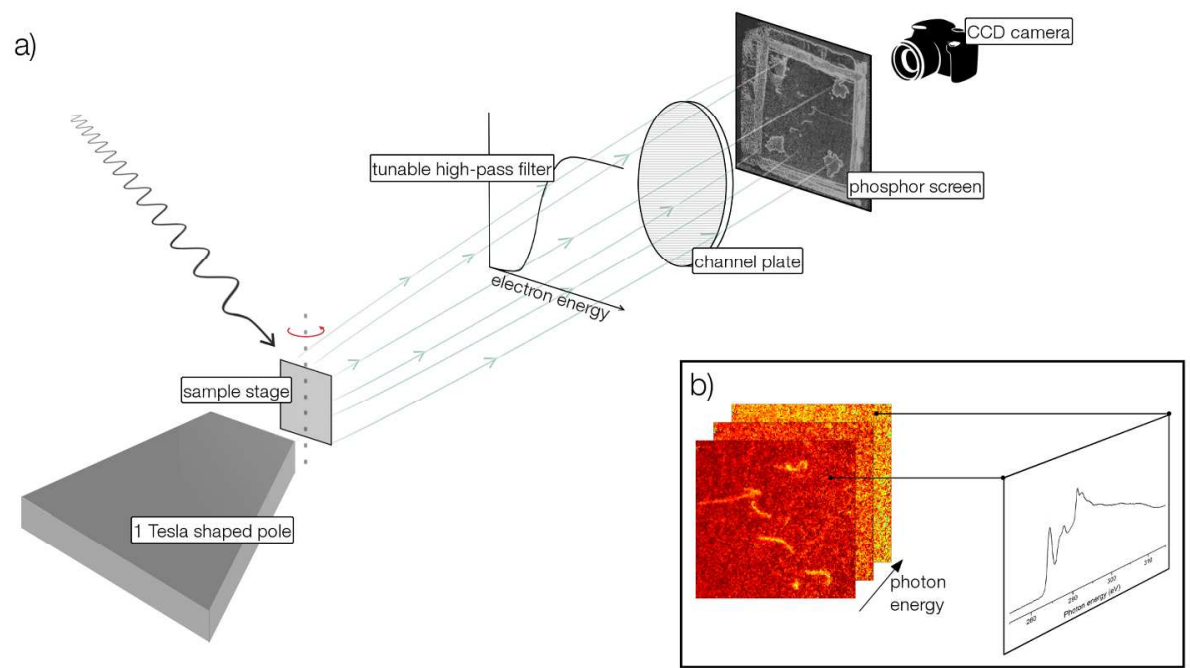

.

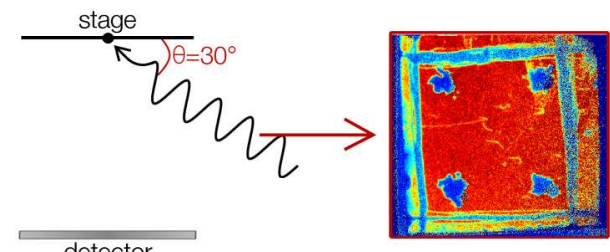

d)
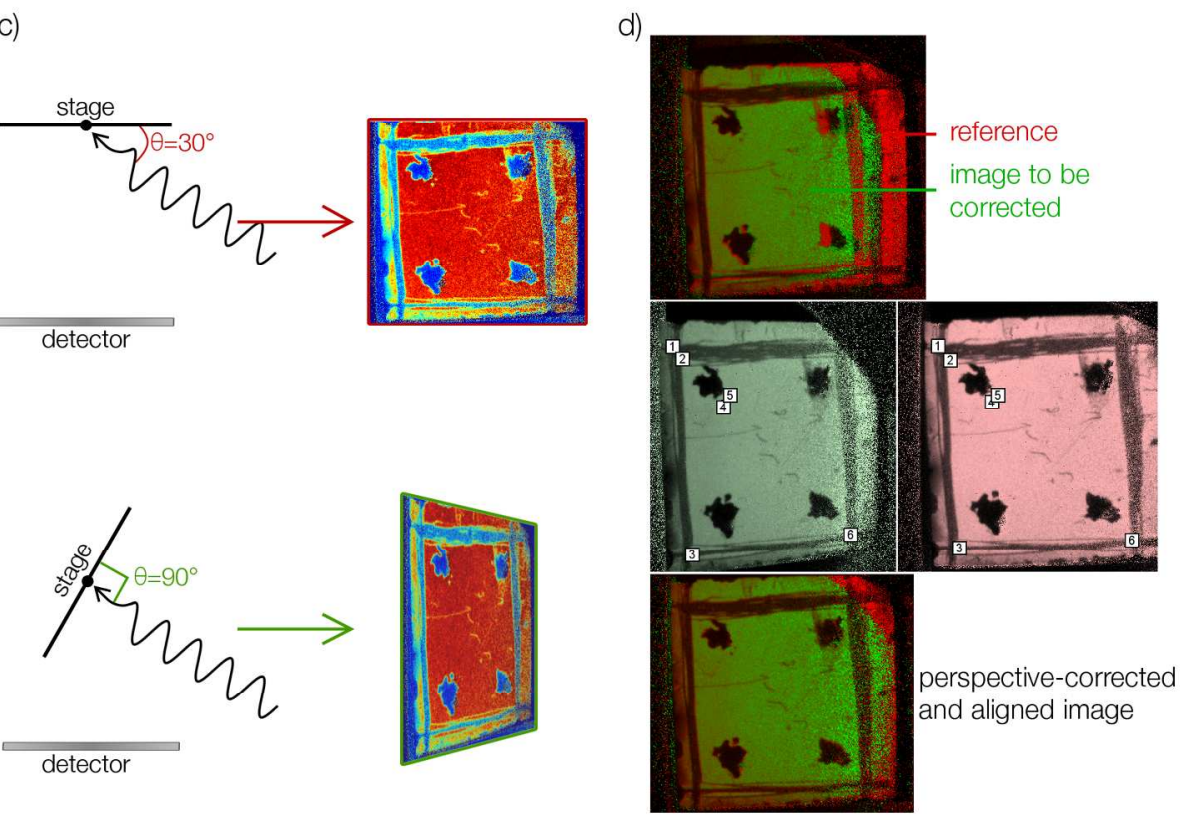

Figure 1. Acquisition of hyperspectral NEXAFS image stacks. a) The sample is submerged in a uniform $1 \mathrm{~T}$ magnetic field which confines electron trajectories. The photon beam is wobbled up and down to provide full-field illumination for each incident photon energy. Emitted Auger electrons travel along the magnetic field lines, through a high-pass filter and a channel plate. Generated electron pulses are impinged on a phosphor-coated fibre-optic feedthrough, which is imaged with a CCD camera. b) The sample (here, epitaxially-grown graphene) is imaged at each photon energy interval-each pixel in the stack represents an individual spectrum. c) Tilting the stage to acquire NEXAFS at various angles results in spatially-uncorrelated stacks. d) Stacks from different angular acquisitions are manually corrected by correlating pixels to a reference using the LDF software. 
a)

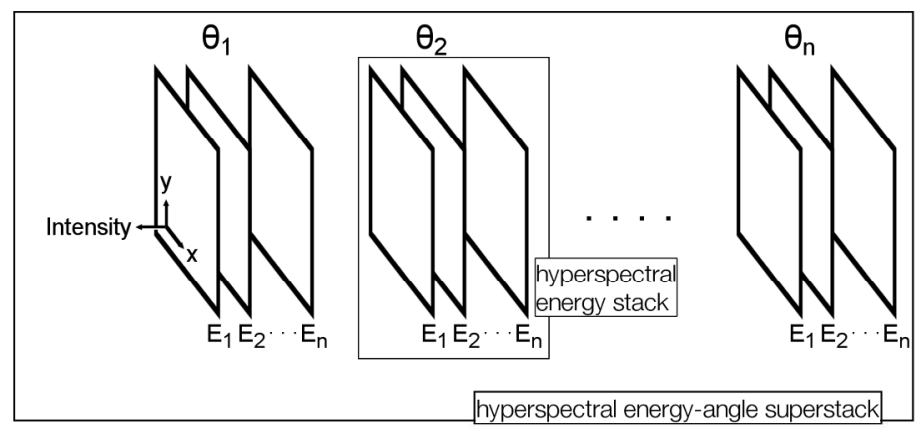

b)
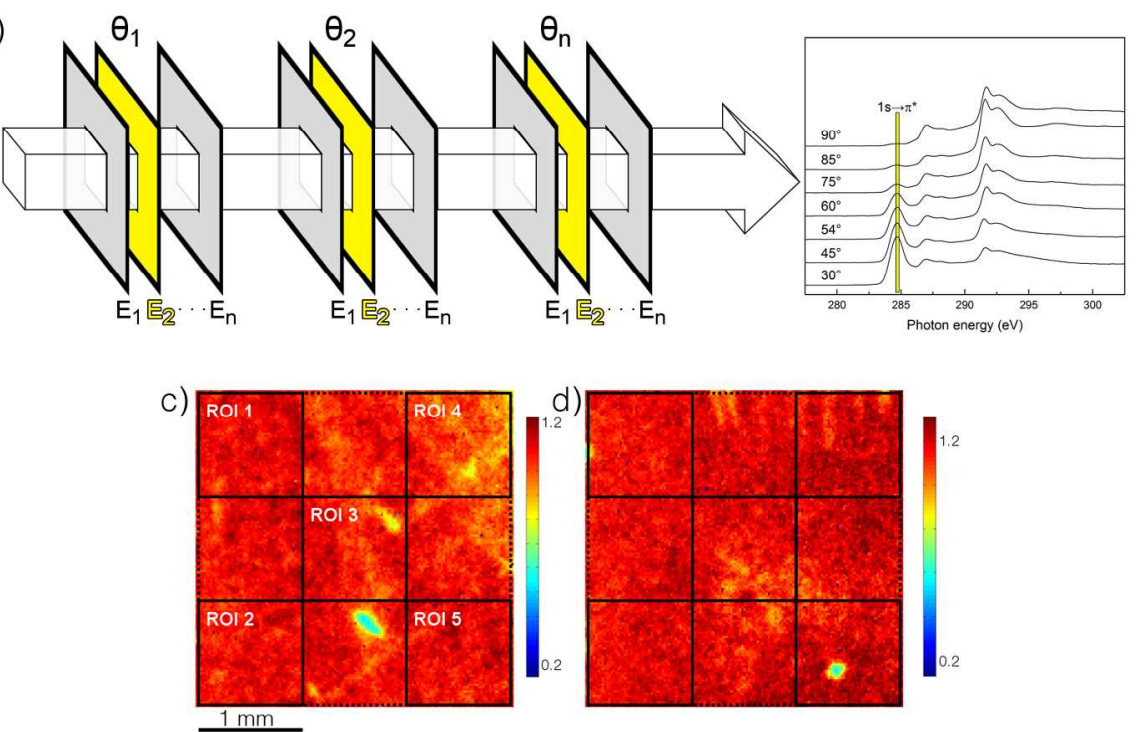

e)
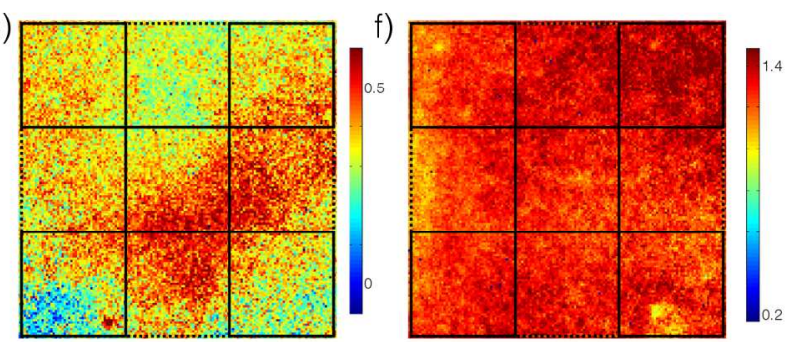

Figure 2. Alignment and perspective correction of experimental data to enable precise ROI correlation between angular acquisitions, and NEXAFS scans of graphene systems under study. a) Post-correction, a hyperspectral energy-angle superstack is assembled for each experimental system, consisting of a hyperspectral energy stack for every acquisition angle. Superstacks are thus functions of spatial $x$ and $y$ coordinates, photon energy, intensity, and acquisition angle. b) Schematic representation of data extraction: angular spectra acquired from a precise area in a graphene system, with energy E2 representing the $\Pi^{*}$ resonance. c) SLG/SiC-Si, d) 4LG/SiC-Si, e) SLG/SiC-C, f) 4LG/SiC-C. Images tuned around 284.7 $\mathrm{eV}$, and acquired at $30^{\circ}$, rendering intense $n^{*}$ resonances. ROIs are $1 \mathrm{~mm} \times 1 \mathrm{~mm}$, and each pixel is $\sim 40$ $\mu \mathrm{m}$. 

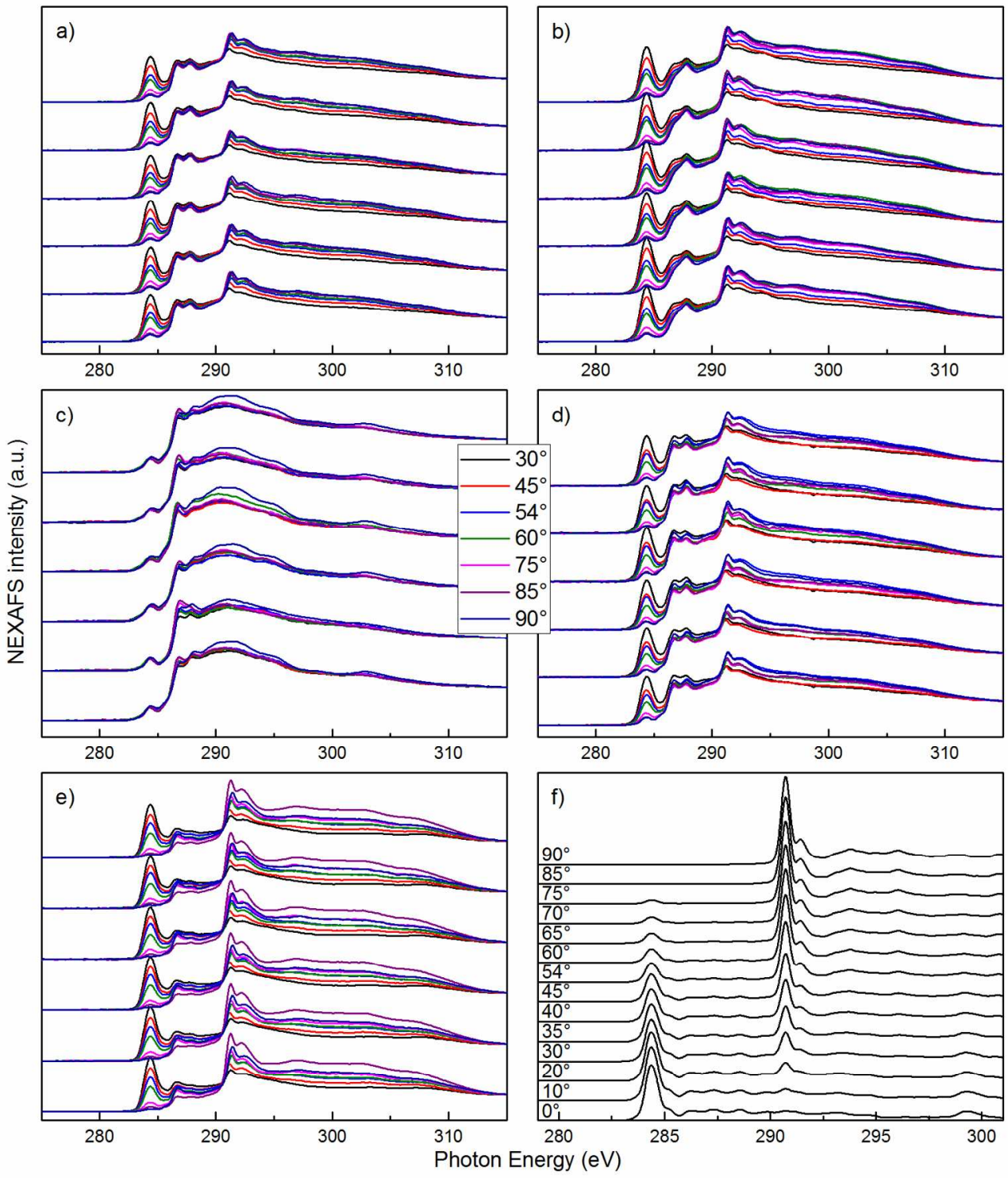

Figure 3. Extracted angle-resolved Carbon K-edge NEXAFS spectra. a) SLG/SiC-Si, b) 4LG/SiC-Si, c) SLG/SiC-C, d) 4LG/SiC-C, e) epitaxial graphene, f) Theoretical freestanding graphene. Resonances described in detail in the Supporting Information. 
a)

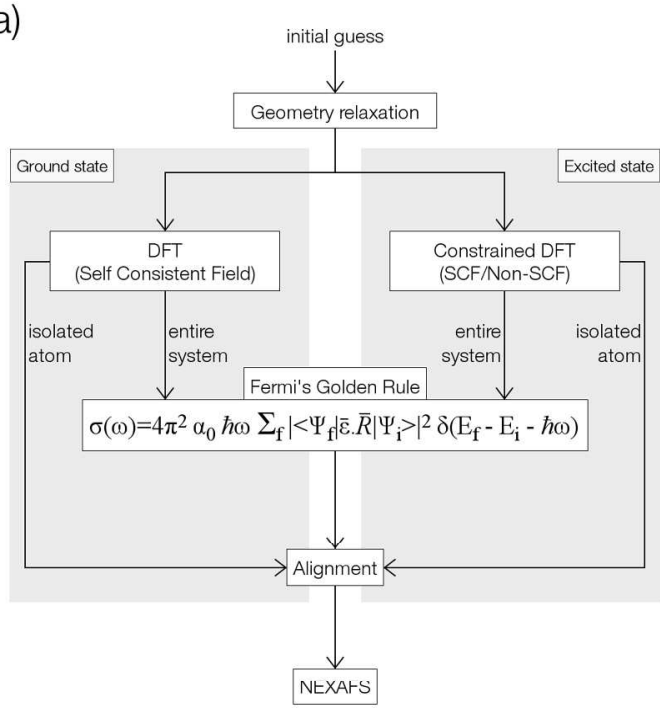

b)

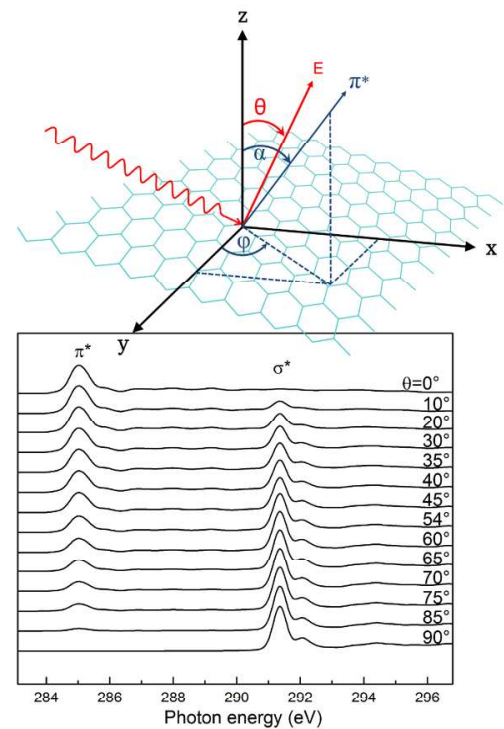

c)

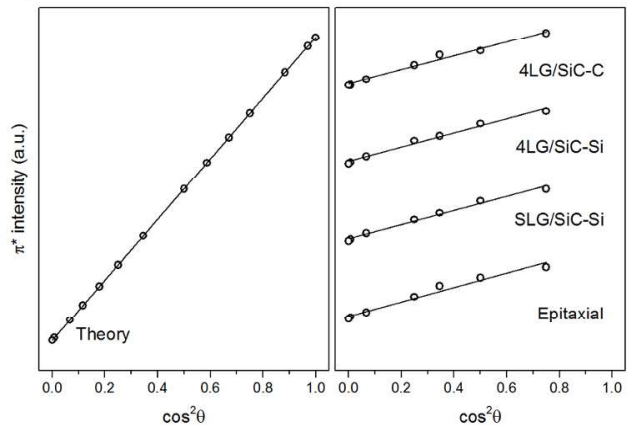

d)

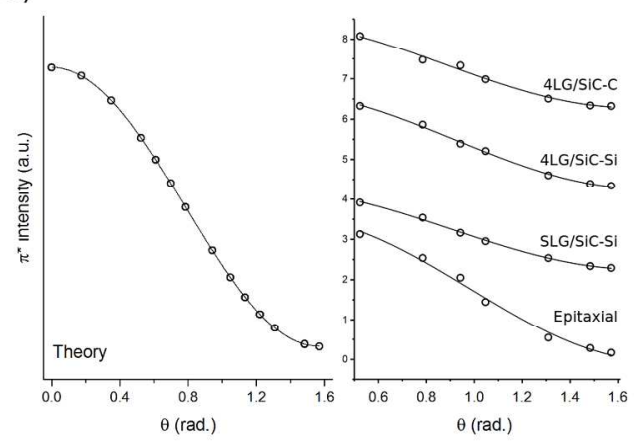

Figure 4. Theoretical angular-resolved NEXAFS calculation algorithm, and $\Pi^{*}$ and $\sigma^{*}$ intensities plotted against $\cos 2 \theta$ and $\sin 2 \theta$ respectively for both theoretical and experimental graphene systems. a) Following geometry optimization to find the most energetically stable structure, the ground state is computed through standard DFT, whilst the excited state is computed through constrained DFT following the excited electron and core hole $(\mathrm{XCH})$ approximation. NEXAFS spectra are calculated using Fermi's Golden Rule. b) Spectra are then projected for various beam polar angles $(\theta)$; geometric definitions also show $\Pi^{*}$ vector described by angles $\mathrm{a}$ and $\varphi$ as used in the orbital vector approximation. c) Dichroic ratios of theoretical graphene, epitaxial graphene, SLG/SiC-Si, 4LG/SiC-Si and 4LG/SiC-C computed from $3 \mathrm{~mm} \times 3 \mathrm{~mm}$ ROIs are -1, $0.93,-0.94,-0.94$, and -0.97 , respectively. Full results presented in Table S1, Supporting Information. d) $\Pi^{*}$ NEXAFS intensities fitted with Eq. 2. Fitting parameter afrom $3 \mathrm{~mm} \times 3 \mathrm{~mm}$ ROIs resulted in 0 for theoretical graphene, 11.8 for epitaxial graphene/SiC, 7.2 for SLG/SiC-Si, 7.9 for $4 \mathrm{LG} / \mathrm{SiC}-\mathrm{Si}$, and 5.5 for $4 \mathrm{LG} / \mathrm{SiC}-\mathrm{C}$. Full results are presented in Table S2, Supporting Information.

$170 \times 162 \mathrm{~mm}(300 \times 300$ DPI $)$ 


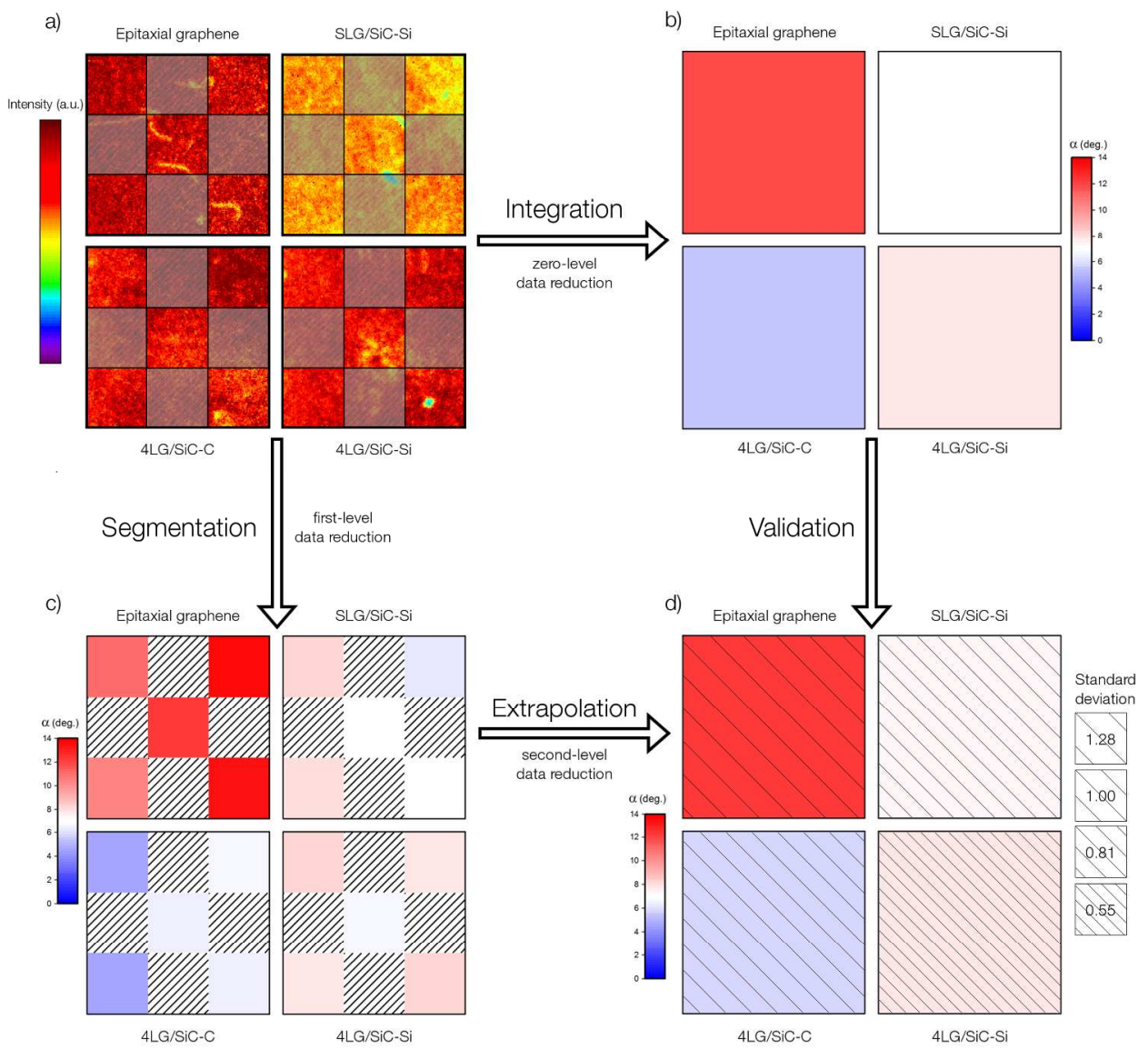

Figure 5. a maps constructed from results of orbital vector approximation. a) NEXAFS $n^{*}$ intensities, b) as from $3 \mathrm{~mm} \times 3 \mathrm{~mm}$ ROIs (zero-level data reduction), c) as from ROIs 1-5 (first-level data reduction), d) mean as and standard deviations from ROIs 1-5 (second-level data reduction). Intensity scale in a) is 0-1.9 for epitaxial graphene/SiC, and 0-1.4 for the other three systems.

$194 \times 172 \mathrm{~mm}(300 \times 300 \mathrm{DPI})$ 


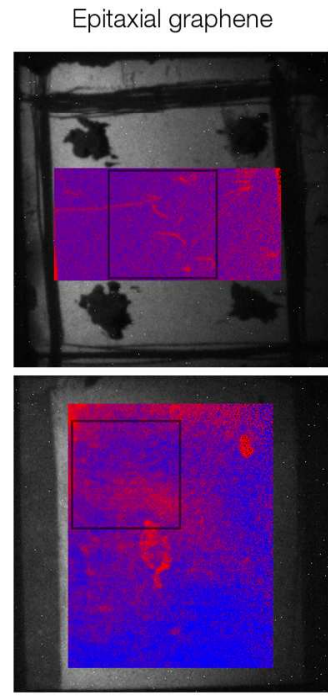

4LG/SiC-C

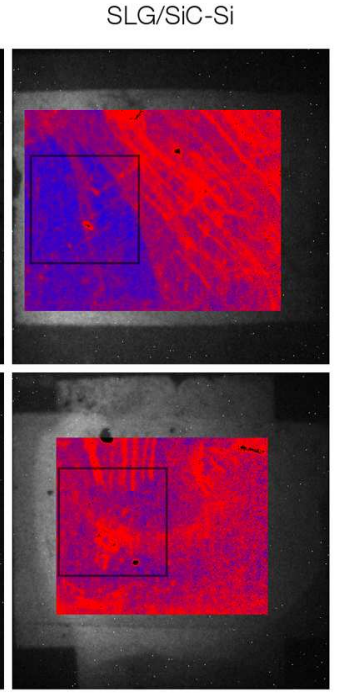

4LG/SiC-Si

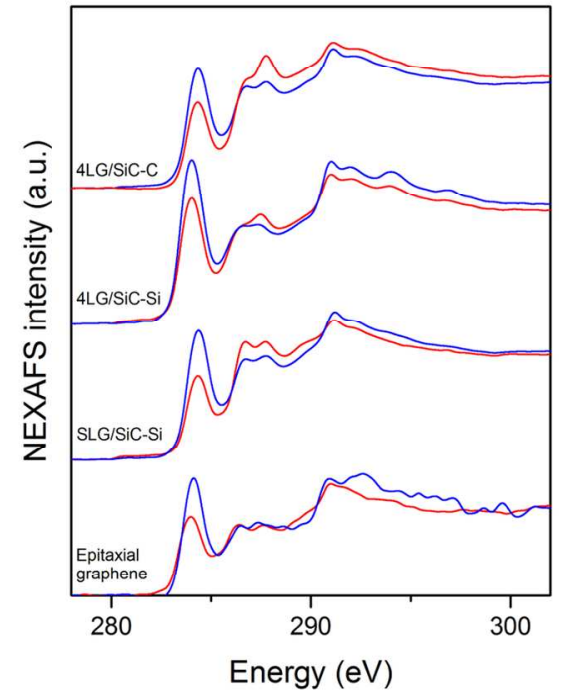

Figure 6. DBSCAN clustering based on $\Pi^{*}$ intensity. Cluster maps indicate 4LG/SiC-C has the most pristine graphene. Maps also suggest two distinct length scales of out-of-plane deformations. Black squares indicate the location of $3 \mathrm{~mm} \times 3 \mathrm{~mm}$ ROIs analyzed in the previous sections. 

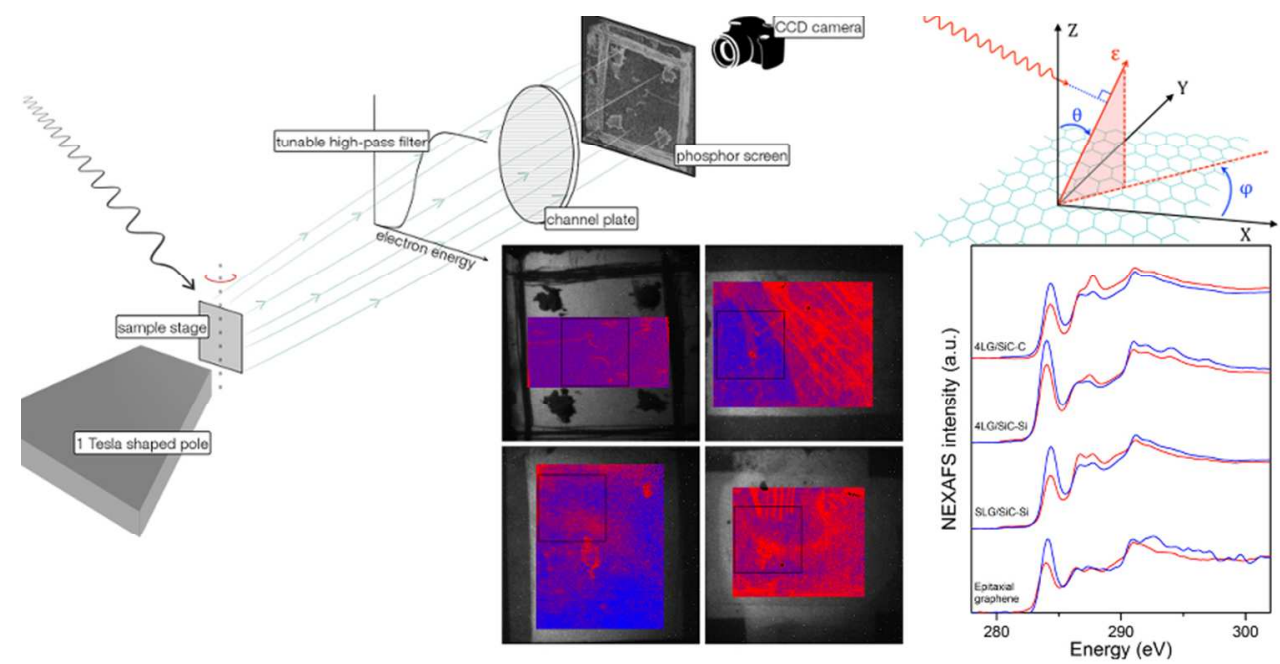

Table of contents

$80 \times 39 \mathrm{~mm}(300 \times 300$ DPI $)$ 